\title{
Oxidative stress-induced misfolding and inclusion formation of Nrf2 and Keap1
}

\author{
Vy Ngo ${ }^{1}$, Nadun C. Karunatilleke ${ }^{2}$, Anne Brickenden, Wing-Yiu Choy ${ }^{2}$ and Martin L. Duennwald ${ }^{3, *}$ \\ 1 Department of Pathology and Laboratory Medicine, Schulich School of Medicine and Dentistry, University \\ of Western Ontario, London, Ontario, Canada; vngo23@uwo.ca (V.N.) \\ 2 Department of Biochemistry, Schulich School of Medicine and Dentistry, University of Western Ontario, \\ London, Ontario, Canada; nkarunat@uwo.ca (N.C.K.); zsong83@uwo.ca (Z.S.); abricken@uwo.ca (A.B.); \\ jchoy4@uwo.ca (W.Y.C.) \\ 3 Department of Anatomy and Cell Biology, Schulich School of Medicine and Dentistry, University of West- \\ ern Ontario, London, Ontario, Canada; martin.duennwald@schulich.uwo.ca (M.L.D.) \\ * Correspondence: martin.duennwald@schulich.uwo.ca
}

\begin{abstract}
Cells that experience high levels of oxidative stress respond with the induction of antioxidant proteins through the activation of the transcription factor Nrf2. Nrf2 is negatively regulated by Keap 1 which binds to Nrf2 to facilitate its ubiquitination and ensuing proteasomal degradation under basal conditions. Here, we study protein folding and misfolding in Nrf2 and Keap1 in yeast, mammalian cells, and purified proteins under oxidative stress conditions. Both Nrf2 and Keap1 are susceptible to protein misfolding and inclusion formation upon oxidative stress. We propose that the intrinsically disordered regions within Nrf2 and the high cysteine content of Keap1 contribute to their oxidation and the ensuing misfolding. Our work reveals previously unexplored aspects of Nrf2 and Keap1 regulation and dysregulation by oxidation-induced protein misfolding.
\end{abstract}

Keywords: Nrf2; Keap1; oxidation; oxidative stress; protein misfolding

\section{Introduction}

Oxidative stress is regulated by the transcription factor nuclear factor erythroid 2related factor 2 (Nrf2) [1]. Nrf2 regulates the expression of a multitude of antioxidant genes and is negatively regulated by Kelch-like ECH-associated protein 1 (Keap1) [2], a substrate adaptor protein that binds to Nrf2 in the cytoplasm to promote its ubiquitination and ensuing degradation by the proteasome [2-6]. During oxidative stress, specific stresssensing cysteine residues in Keap1 become oxidized [7-9], resulting in a conformational change in Keap1 that leads to dissociation of the Keap1-Nrf2 complex. This, in turn, leads to Nrf2 stabilization, nuclear translocation, and ultimately, activation of cytoprotective antioxidant genes [10,11]. Induction of the Keap1-Nrf2 antioxidant pathway is fundamental to protecting cells against oxidative stress, and mutations that disrupt Keap1-Nrf2 binding compromise Nrf2 regulation and can contribute to numerous human diseases [12, 13]. The transcriptional activity of Nrf2 is therefore tightly regulated by its interaction with Keap1 [14, 15].

Nrf2 contains seven conserved regions that are referred to as the Nrf2-ECH homology (Neh) domains. Notably, the Neh2 domain that mediates Nrf2's binding with Keap1 is mostly intrinsically disordered [14], and transiently structured elements within the Neh2, Neh7, and Neh1 domains of Nrf2 render the protein partially intrinsically disordered [16]. Intrinsically disordered proteins (IDPs) or proteins that contain long intrinsically disordered regions (IDRs) lack a fixed three-dimensional structure and are susceptible to protein misfolding and inclusion formation in cells due to their structural heterogeneity and flexible nature; however, this also allows for enhanced binding capacity and multifunctionality [17-19]. This may explain Nrf2's ability to bind to a vast array of different proteins. We use the term 'protein misfolding' here to indicate proteins that have 
acquired a non-native, aberrant conformation, often in the form of inclusions or aggregates. Misfolded proteins often lose their normal function (i.e., loss of protein function) and tend to aggregate and form inclusions that can have deleterious effects on the cell (i.e., a toxic gain of function) [20]. Examples of disease-associated IDPs include $\alpha$-synuclein in Parkinson's disease [21], while in cancer, many key tumour suppressors contain long IDRs, such as p53 [22]. Interestingly, oxidative stress can affect the structural flexibility of IDPs/IDRs [21] and may thus, also modulate the folding or contribute to the misfolding of intrinsically disordered Nrf2, which may impair or alter its interactions with other proteins and thus, its transcriptional activity.

Protein misfolding may also expose hydrophobic or oxidation-prone cysteine residues to the surface of the protein, rendering them targets for oxidation by reactive oxygen species (ROS) and other oxidants [23]. Oxidation products of cysteines include disulfide bonds and mixed disulfide bonds. Oxidation can also lead to alteration of non-covalent interactions within proteins, fragmentation of peptide chains, cross-linking of proteins, and/or oxidation of specific side chains, ultimately leading to protein destabilization and misfolding [24-26]. Cysteine residues are particularly susceptible to aberrant oxidation by ROS due to the presence of their nucleophilic thiol groups [27]. Keap1, which contains an exceptionally high percentage of cysteine residues, may thus be particularly susceptible to oxidation and inclusion formation.

In this study, we examine two aspects of protein oxidation: the misfolding of the intrinsically disordered Nrf2, and the misfolding of the cysteine-rich Keap1, under oxidative stress conditions. Using yeast, cultured mammalian cells, and purified proteins, we find that both Nrf2 and Keap1 misfold and form aberrant cytoplasmic and possibly nuclear protein inclusions upon exposure to high levels of oxidative stress. Our results suggest a previously unexplored mechanism by which the Keap1-Nrf2 interaction may be altered by oxidative stress as it pertains to protein misfolding and inclusion formation.

\section{Materials and Methods}

\subsection{Prediction of intrinsically disordered regions}

Three independent algorithms, PrDOS, IUPred2A, and PONDR [28-30] were used to predict intrinsically disordered regions using protein sequences obtained from UniProt [31]. Using the scores obtained from each algorithm, each amino acid residue within the protein was assigned a numerical value of ' +1 ' or ' -1 ', where $>0.5=+1$ (disordered) and values $<0.5=-1$ (ordered). The scores were summed for each residue. Residues with a combined score of +3 across all three algorithms were considered disordered.

\subsection{Protein sequence alignment}

MEGA X [32] was used to perform protein sequence alignments using protein sequences obtained from UniProt [31].

\subsection{Yeast growth assays and microscopy}

For assessment of relative growth, wild-type yeast and deletion strains obtained from the Saccharomyces Genome Deletion Project [33] were used. Yeast cells were transformed using the standard lithium acetate/salmon sperm carrier DNA/PEG method for the incorporation of yeast plasmids [34]. Transformed yeast cells were grown overnight in synthetic selective media to maintain these plasmids. Growth assays were performed by spotting $5 \mathrm{X}$ serial dilutions of $\mathrm{OD}_{600}=0.2$ on agar plates and incubated at $30^{\circ} \mathrm{C}$. Plates were imaged using the Bio-Rad ChemiDoc (Bio-Rad). Growth was quantified as previously described [35]. For assessment of protein expression using fluorescence microscopy, yeast was transformed with YFP-tagged plasmids. Cells were transferred to a glass microscope slide and coverslip and imaged using the Olympus BX-51 Bright Field/Fluorescence Microscope at 60X and captured using an equipped CCD camera (Spot Pursuit).

\subsection{Cell lines and culture conditions}


The HeLa cell line (human, cervical cancer cell line) was maintained in Dulbecco's Modified Eagle Medium (DMEM; Gibco, 41966-029), supplemented with 10\% FBS (Wisent, 080-150) and $1 \mathrm{X}$ penicillin-streptomycin (Corning, 30-001-CI). Cells were cultured at $37^{\circ} \mathrm{C}$ with $5 \% \mathrm{CO}_{2}$. For transfections, cells were seeded in a 6 -well plate at $1.0 \times 10^{6}$ cells per well and grown to approximately $80 \%$ confluency. Cells were transfected with Lipofectamine LTX with PLUS Reagent (Thermo Fisher Scientific, A12621) according to the manufacturer's protocol in Opti-MEM I Reduced Serum Medium (Gibco, 31985-062). Transfected cells were incubated at $37^{\circ} \mathrm{C}$ for $6 \mathrm{~h}$, followed by a wash in $1 \mathrm{X}$ PBS, and incubated in DMEM for $18 \mathrm{~h}$ at $37^{\circ} \mathrm{C}$. Cells were then split into the appropriate plates for subsequent experiments.

\subsection{Cell viability assays}

To determine the optimal hydrogen peroxide treatment concentration and duration, transfected HeLa cells were seeded into 96-well solid white microplates (Greiner, M018732EA) at $4 \times 10^{4}$ cells per well and incubated for $16 \mathrm{~h}$. Following treatment, cell viability was assessed using the CellTiter-Glo 2.0 Cell Viability Assay (Promega, G9242) according to the manufacturer's protocol. Luminescence was measured using the Cytation 5 Cell Imaging Multi-Mode Reader (BioTek).

\subsection{Fluorescence and immunofluorescence microscopy}

Transfected HeLa cells were seeded on a $15 \mathrm{~mm}$ circular glass coverslip (Matsunami, C015001) in a 12-well plate at $1 \times 10^{5}$ to ensure approximately $75 \%$ confluency the following day. Cells were fixed with $4 \%$ paraformaldehyde, permeabilized with $0.1 \%$ Triton X-100 in PBS, blocked with $20 \%$ goat head serum in PBB (0.5\% BSA in PBS), and incubated with one of the following primary antibodies overnight at $4^{\circ} \mathrm{C}$ at a concentration of 1:100: mouse anti-Nrf2 (Abcam, ab62352) or mouse anti-Keap1 (Proteintech, 10503-2-AP). The coverslips were incubated with the following Alexa Fluor 488-conjugated secondary antibody for $1 \mathrm{~h}$ at room temperature at a concentration of 1:1500: goat anti-mouse (Thermo Fisher Scientific, A-11094). Coverslips were mounted onto glass microscope slides with SlowFade Gold Antifade Reagent with DAPI (Thermo Fisher Scientific, S36938) and cured at room temperature for $24 \mathrm{~h}$. Cells were imaged using the Cytation 5 Cell Imaging MultiMode Reader (BioTek) using a 20X objective lens, or the Nikon Ti2-E Inverted Microscope paired with the A1R HD laser-scanning confocal (Nikon) using a 20x or 40x objective lens.

\section{7. $R N A$ isolation and quantitative reverse transcription $P C R$ (RT-qPCR)}

HeLa cells were seeded in a 6-well plate at $1 \times 10^{6}$ cells per well, grown to $~ 85 \%$ confluency, and treated with $300 \mu \mathrm{l}$ of $\mathrm{H}_{2} \mathrm{O}_{2}$ for $3 \mathrm{~h}$. RNA was isolated using the RNA Isolation Kit (AdvanTech Genomic, AD100-31701), and 500 ng of RNA was converted into cDNA using the RevertAid H Minus First Strand cDNA Synthesis Kit (Thermo Fisher Scientific, K1632). The PowerUp SYBR Green Master Mix (Applied Biosystems, A25742) was utilized for quantitative PCR with the primer sequences listed in Error! Reference source not found.. The output values were normalized to RPLPO expression.

Table 1. Primer sequences utilized for RT-qPCR.

\begin{tabular}{cl}
\hline mRNA Probe & $\begin{array}{l}\text { Primer Sequences } \\
\left.\text { (Forward and Reverse, } 5^{\prime} \text { to } 3^{\prime}\right)\end{array}$ \\
\hline NFE2L2 & F: GCCCAATGTGAGAACACACC \\
\hline
\end{tabular}


R: TGTGAGATGAGCCTCCAAGC

$\begin{array}{ll}\text { HMOX1 } & \text { F: CCCCAACGAAAAGCACATCC } \\ & \text { R: AGACAGCTGCCACATTAGGG } \\ \text { NQO1 } & \text { F: TGGAAGAAACGCCTGGAGAAT } \\ & \text { R: CTGGTTGTCAGTTGGGATGG } \\ \text { TXN } & \text { F: ATTGTGACCAGCACCTACGG } \\ & \text { R: CATGGTGGAGTTGCCCGAA } \\ \text { RPLP0 } & \text { F: CCTCATATCCGGGGGAATGTG } \\ & \text { R: GCAGCAGCTGGCACCTTATTG }\end{array}$

\subsection{SDS-PAGE and Coomassie blue gel staining}

Purified protein $(10 \mu \mathrm{g})$ was resolved on a $10 \%$ SDS-PAGE gel. The gel was stained with Coomassie Brilliant Blue (0.1\% Coomassie Brilliant Blue R-250, 50\% methanol [v/v], $10 \%$ glacial acetic acid $[\mathrm{v} / \mathrm{v}], 40 \% \mathrm{H}_{2} \mathrm{O}$ ) for 30 minutes and de-stained overnight using a destining solution $\left(50 \%\right.$ methanol $[\mathrm{v} / \mathrm{v}], 10 \%$ glacial acetic acid $\left.[\mathrm{v} / \mathrm{v}], 40 \% \mathrm{H}_{2} \mathrm{O}\right)$ with gentle agitation. Blots were imaged using the ChemiDoc Imaging System (Bio-Rad).

\subsection{SDD-AGE (semi-denaturing detergent agarose gel electrophoresis)}

Purified protein $(10 \mu \mathrm{g})$ was resolved on a $1.8 \%$ agaraose-2\% SDS gel and run at 80 $\mathrm{V}$ for approximately $1.5 \mathrm{~h}$ at room temperature in $1 \mathrm{X}$ TAE- $0.1 \%$ SDS running buffer. The gel was transferred at room temperature to PVDF by an overnight wet transfer by gravity according to the manufacturer's protocol (Whatman TurboBlotter Transfer System). The membrane was blocked with 5\% skim milk in PBST (1X PBS, 1\% Tween-20) and incubated with one of the following primary antibodies overnight at $4^{\circ} \mathrm{C}$ at a concentration of 1:1000: mouse anti-Nrf2 (Abcam, ab62352) or mouse anti-Keap1 (Proteintech, 10503-2-AP). The membrane was incubated with the following Alexa Fluor 680-conjugated antibody for $1 \mathrm{~h}$ at room temperature at a concentration of 1:1500: goat anti-mouse (Thermo Fisher Scientific, A-21057). Blots were imaged using the ChemiDoc Imaging System (Bio-Rad).

\subsection{Combined SDD-AGE and fractionation assay}

For partitioning into soluble and insoluble fractions, purified protein was first aliquoted into a chilled microcentrifuge tube which represents the 'total' fraction. A second aliquot was centrifuged at $10000 \mathrm{xg}$ for $10 \mathrm{~min}$ at $4^{\circ} \mathrm{C}$. The supernatant was transferred into a chilled microcentrifuge tube which represented the soluble 'supernatant' fraction. The pellet was resuspended in dialysis buffer (from the preceding protein purification process) and represented the insoluble 'pellet' fraction. Equal volumes of each fraction equivalent to $10 \mu \mathrm{g}$ of the total fraction were resolved by SDD-AGE (see above).

\subsection{Statistical analysis}

Statistical analyses were conducted using Prism 8 (GraphPad Software). Statistical significance was obtained by performing a one-way ANOVA with Tukey post hoc for comparison between groups. Error bars represent standard deviation. P-values less than 0.05 were considered statistically significant. Significance levels are indicated using asterisks, where ${ }^{*}$ is $\mathrm{p}<0.05,{ }^{* *}$ is $\mathrm{p}<0.01$, and ${ }^{* * *}$ is $\mathrm{p}<0.001$. Shapiro-Wilk tests were performed for all data sets to ensure normality.

\section{Results}




\subsection{Analysis of Nrf2}

3.1.1. Nrf2 is intrinsically disordered and Keap1's high cysteine content is evolutionarily conserved

Figure 1A schematically illustrates the functional domains of human Nrf2 and Keap1. Nrf2 contains seven conserved Neh domains and six cysteine residues, whereas Keap1 contains three functional domains and a total of 27 cysteine residues. The key oxidative stress-sensing sensor cysteines in Keap1 are indicated with an asterisk $\left(^{*}\right)$ [7-9, 36].

Disordered profile plots for Nrf2 and Keap1 indicate the location of intrinsically disordered regions as predicted by three independent algorithms (PrDOS, IUPred2A, and PONDR) [28-30] (Figure 1B) (refer to Figure S1 for the individual algorithm predictions). Intrinsically disordered regions are highlighted in yellow. In our analyses, an amino acid residue was denoted as disordered if it was predicted as disordered by all three individual algorithms, i.e., a prediction value of $>0.5=+1$ (disorder) and prediction value of $<0.5=-1$ (order); thus, a combined value of +3 was denoted as disordered. From these results, Nrf2 was predicted to contain eleven intrinsically disordered regions with an overall combined percent disordered score of $39.34 \%$. Our previous work also provides experimental evidence for the highly intrinsically disordered properties of Nrf2 [16]. In comparison, Keap1 is mostly ordered with a single predicted disordered region and an overall combined percent disordered score of $0.48 \%$, which is also corroborated by structural studies of the folded Kelch domain of Keap1 [37].

Next, we analyzed the amino acid composition of Nrf2 and Keap1 and calculated the percentage of cysteine content across 15 metazoan species (Table S1) from humans to zebrafish (Figure 1C). The total cysteine content in human Nrf2 was $0.99 \%$, which is below the average for the human proteome of $2.3 \%$ [38], while the average for Nrf2 across all 15 species analyzed here was $1.11 \%$. Intriguingly, human Keap1 contains has a high $4.33 \%$ cysteine content, which is highly almost double the human average. The overall average for Keap 1 across all species was $4.07 \%$, which demonstrates that the high cysteine content in Keap1 is conserved, even beyond the key cysteines that directly regulate Nrf2 interaction. To determine if cysteine residues are evolutionarily conserved regarding their positions within Keap1 and Nrf2 across the 15 species, we performed a protein sequence alignment using MEGA X [32] and found that all six cysteine residues in Nrf2 (highlighted in yellow) were either completely or highly conserved across species (i.e., perfectly aligned in 13-15 species) (Figure 1D, top). For Keap1, 24 of the 27 cysteine residues in human Keap1 were either completely or highly conserved (i.e., perfectly aligned in 12 species) (Figure 1D, bottom). Unsurprisingly, all sensor cysteines within Keap1 (indicated with an asterisk $\left(^{*}\right)$ ) were also either completely or almost completely conserved (i.e., perfectly aligned in 14 species). 
(A)

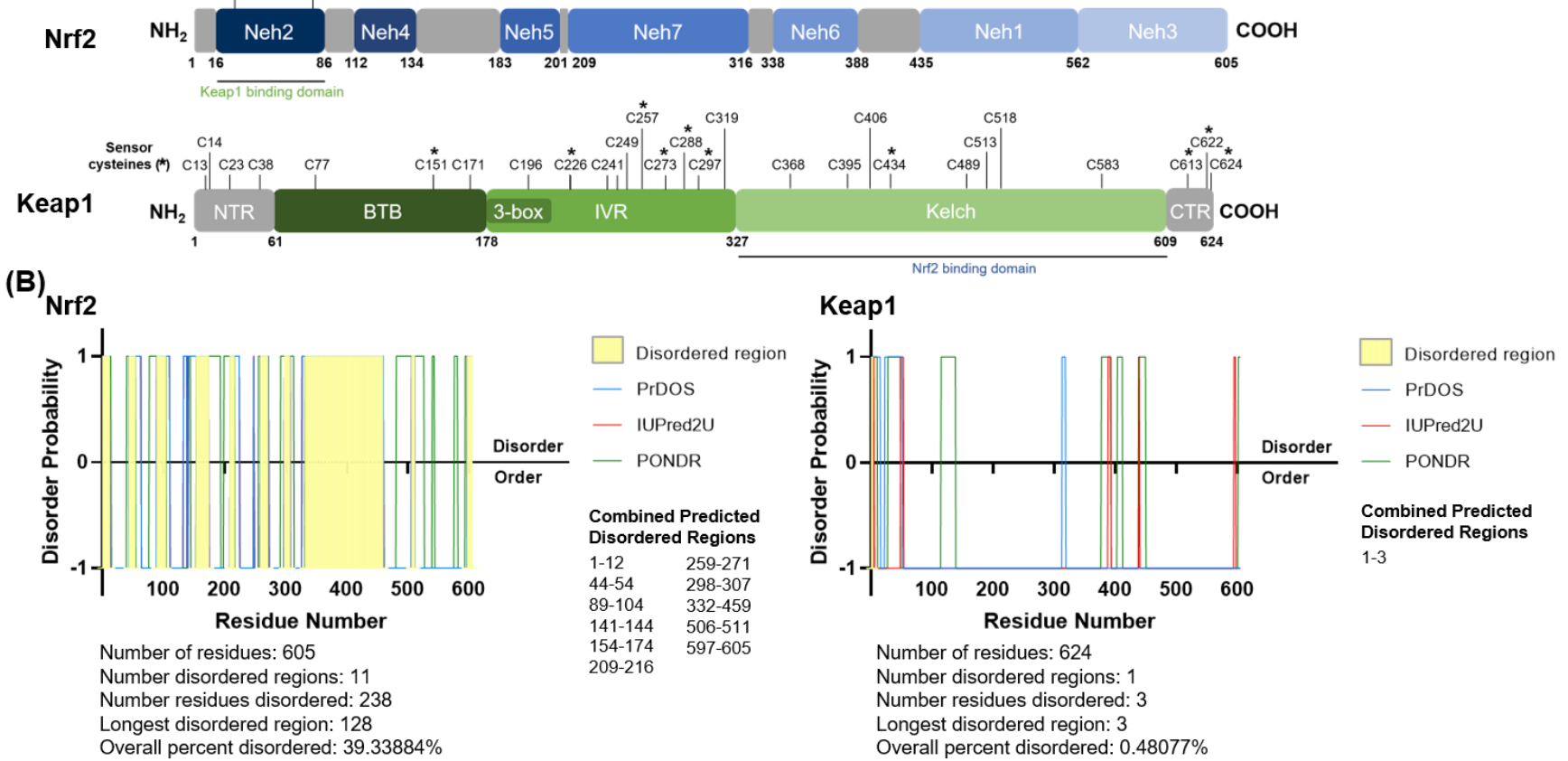

(C) Nrf2

\begin{tabular}{llr}
\hline Species & Specific Name & \% Cys \\
\hline Human & Homo sapiens & 0.99174 \\
Chimpanzee & Pan troglodytes & 1.16667 \\
Orangutan & Pongo abelii & 0.99010 \\
Rhesus macaque & Macaca mulatta & 0.99010 \\
Marmoset & Callithrix jacchus & 0.99010 \\
Galago & Otolemur garnettii & 1.15512 \\
Mouse & Mus musculus & 1.17253 \\
Rat & Rattus norvegicus & 1.15894 \\
Golden hamster & Mesocricetus auratus & 1.16861 \\
Rabbit & Oryctolagus cuniculus & 0.98847 \\
Cow & Bos taurus & 1.32670 \\
Bat & Myotis lucifugus & 0.98847 \\
Elephant & Loxodonta africana & 1.54639 \\
Chicken & Gallus gallus & 1.15894 \\
Zebrafish & Danio rerio & 0.85324 \\
\hline Average \% Cys content in Nrf2 & $\mathbf{1 . 1 0 8 8 9}$ \\
\hline \multicolumn{2}{l}{}
\end{tabular}

\begin{tabular}{llr} 
Keap1 & \\
\hline Species & Specific Name & \% Cys \\
\hline Human & Homo sapiens & 4.32692 \\
Chimpanzee & Pan troglodytes & 4.32692 \\
Orangutan & Pongo abelii & 4.16667 \\
Rhesus macaque & Macaca mulatta & 4.00641 \\
Marmoset & Callithrix jacchus & 4.16667 \\
Galago & Otolemur garnettii & 4.16667 \\
Mouse & Mus musculus & 4.00641 \\
Rat & Rattus norvegicus & 4.00641 \\
Golden hamster & Mesocricetus auratus & 4.00641 \\
Rabbit & Oryctolagus cuniculus & 4.00641 \\
Cow & Bos taurus & 3.84615 \\
Bat & Myotis lucifugus & 4.00641 \\
Elephant & Loxodonta africana & 4.00641 \\
Chicken & Gallus gallus & 4.02010 \\
Zebrafish & Danio rerio & 3.99334 \\
\hline Average \% Cys content in Keap1 & $\mathbf{4 . 0 7 0 8 9}$ \\
\hline \multicolumn{2}{l}{}
\end{tabular}

(D) Residue \# in Human Nrf2 119199242325422514

\begin{tabular}{lcccccc} 
Human & C & C & C & C & C & C \\
Chimpanzee & C & C & C & C & C & C \\
Orangutan & C & C & C & C & C & C \\
Rhesus macaque & C & C & C & C & C & C \\
Marmoset & C & C & C & C & C & C \\
Galago & C & C & C & C & C & C \\
Mouse & C & C & C & C & C & C \\
Rat & C & C & C & C & C & C \\
Golden hamster & C & C & C & C & C & C \\
Rabbit & C & C & C & C & C & C \\
Cow & C & C & C & C & C & C \\
Bat & C & C & C & C & C & C \\
Elephant & C & C & C & C & C & C \\
Chicken & C & C & C & W & R & C \\
Zebrafish & C & C & C & G & L & C \\
\hline
\end{tabular}

\begin{tabular}{|c|c|c|c|c|c|c|c|c|c|c|c|c|c|c|c|c|c|c|c|c|c|c|c|c|c|c|c|}
\hline Residue \# in Human Keap & 13 & 14 & 23 & 38 & 77 & 151 & 171 & 196 & 226 & 241 & 249 & 257 & 273 & 288 & 297 & 319 & 368 & 395 & 406 & 434 & 489 & 513 & 518 & 583 & 613 & 622 & 2624 \\
\hline Key sensor cysteines $\left({ }^{*}\right)$ & & & & & & * & & & $\approx$ & & & * & * & * & $*$ & & & & & $\approx$ & & & & & * & * & * \\
\hline Human & $\mathrm{C}$ & $\mathrm{C}$ & $\mathrm{C}$ & $\mathrm{C}$ & $\mathrm{C}$ & $\mathrm{C}$ & $\mathrm{C}$ & $\mathrm{C}$ & $\mathrm{C}$ & $\mathrm{C}$ & $\mathrm{C}$ & C & $\mathrm{C}$ & $\mathrm{C}$ & $\mathrm{C}$ & C & $\mathrm{C}$ & $\mathrm{C}$ & $\mathrm{C}$ & $\mathrm{C}$ & $\mathrm{C}$ & C & $\mathrm{C}$ & $\mathrm{C}$ & $\mathrm{C}$ & $\mathrm{C}$ & $\mathrm{C}$ \\
\hline Chimpanzee & $\mathrm{C}$ & $\mathrm{C}$ & $\mathrm{C}$ & C & $\mathrm{C}$ & C & C & C & C & C & $\mathrm{C}$ & $\mathrm{C}$ & $\mathrm{C}$ & C & C & C & C & C & C & C & C & $\mathrm{C}$ & $\mathrm{C}$ & $\mathrm{C}$ & $\mathrm{C}$ & $\mathrm{C}$ & C \\
\hline Orangutan & $\mathrm{C}$ & $\mathrm{s}$ & $\mathrm{C}$ & $\mathrm{c}$ & $\mathrm{c}$ & $\mathrm{C}$ & $\mathrm{c}$ & $\mathrm{c}$ & $\mathrm{c}$ & $\mathrm{c}$ & $\mathrm{c}$ & $\mathrm{c}$ & $\mathrm{c}$ & $\mathrm{c}$ & $\mathrm{c}$ & c & C & C & $\mathrm{c}$ & $\mathrm{c}$ & $\mathrm{C}$ & $\mathrm{c}$ & $\mathrm{c}$ & c & $\mathrm{c}$ & $\mathrm{C}$ & $\mathrm{C}$ \\
\hline Rhesus macaque & C & $\mathrm{s}$ & C & C & C & C & C & C & C & C & C & C & C & C & C & C & C & C & C & C & $\mathrm{R}$ & C & C & C & C & C & C \\
\hline Marmose & $\mathrm{c}$ & $\mathrm{s}$ & $\mathrm{c}$ & $\mathrm{c}$ & C & C & $\mathrm{c}$ & $\mathrm{c}$ & C & $\mathrm{c}$ & $\mathrm{c}$ & $\mathrm{c}$ & $\mathrm{c}$ & $\mathrm{c}$ & c & $\mathrm{c}$ & C & $\mathrm{c}$ & $\mathrm{c}$ & c & C & $\mathrm{c}$ & c & $\mathrm{c}$ & $\mathrm{C}$ & $\mathrm{c}$ & c \\
\hline Galago & $\mathrm{R}$ & $\mathrm{s}$ & C & $\mathrm{C}$ & $\mathrm{C}$ & C & $\mathrm{C}$ & C & $\mathrm{C}$ & c & $\mathrm{C}$ & C & $\mathrm{C}$ & C & C & C & C & C & C & C & C & C & $\mathrm{C}$ & $\mathrm{C}$ & $\mathrm{C}$ & $\mathrm{C}$ & C \\
\hline Mouse & $\mathrm{s}$ & $\mathrm{s}$ & $\mathrm{C}$ & $\mathrm{c}$ & $\mathrm{c}$ & $\mathrm{C}$ & $\mathrm{c}$ & $\mathrm{c}$ & $\mathrm{C}$ & $\mathrm{c}$ & $\mathrm{c}$ & $\mathrm{c}$ & $\mathrm{c}$ & $\mathrm{c}$ & c & c & C & c & $\mathrm{c}$ & c & c & $\mathrm{c}$ & $\mathrm{C}$ & $\mathrm{c}$ & $\mathrm{c}$ & c & c \\
\hline Rat & s & s & $\mathrm{c}$ & $\mathrm{c}$ & $\mathrm{c}$ & $\mathrm{C}$ & c & $\mathrm{c}$ & $\mathrm{c}$ & c & $\mathrm{c}$ & $\mathrm{c}$ & $\mathrm{c}$ & $\mathrm{c}$ & $\mathrm{c}$ & c & $\mathrm{C}$ & C & c & C & $\mathrm{C}$ & c & $\mathrm{C}$ & C & $\mathrm{c}$ & $\mathrm{C}$ & $\mathrm{C}$ \\
\hline Golden hamster & $\mathrm{s}$ & $\mathrm{s}$ & $\mathrm{c}$ & $\mathrm{c}$ & $\mathrm{c}$ & $\mathrm{C}$ & $\mathrm{c}$ & $\mathrm{c}$ & $\mathrm{C}$ & $\mathrm{c}$ & $\mathrm{C}$ & $\mathrm{c}$ & $\mathrm{C}$ & $\mathrm{c}$ & $\mathrm{c}$ & $\mathrm{c}$ & C & $\mathrm{c}$ & $\mathrm{c}$ & $\mathrm{c}$ & $\mathrm{C}$ & $\mathrm{c}$ & $\mathrm{C}$ & $\mathrm{c}$ & $\mathrm{c}$ & $\mathrm{C}$ & $\mathrm{c}$ \\
\hline Rabbit & $\mathrm{C}$ & $\mathrm{s}$ & $\mathrm{R}$ & $\mathrm{C}$ & $\mathrm{C}$ & C & $\mathrm{C}$ & $\mathrm{C}$ & $\mathrm{C}$ & $\mathrm{C}$ & $\mathrm{C}$ & c & $\mathrm{C}$ & $\mathrm{C}$ & $\mathrm{C}$ & C & C & $\mathrm{c}$ & $\mathrm{C}$ & $\mathrm{C}$ & $\mathrm{C}$ & $\mathrm{C}$ & $\mathrm{C}$ & $\mathrm{C}$ & $\mathrm{C}$ & $\mathrm{C}$ & $\mathrm{C}$ \\
\hline Cow & $\mathrm{H}$ & $\mathrm{T}$ & $\mathrm{R}$ & $\mathrm{c}$ & $\mathrm{c}$ & $\mathrm{c}$ & $\mathrm{c}$ & $\mathrm{c}$ & $\mathrm{C}$ & $\mathrm{c}$ & $\mathrm{c}$ & $\mathrm{c}$ & $\mathrm{C}$ & $\mathrm{c}$ & c & $\mathrm{c}$ & C & $\mathrm{c}$ & $\mathrm{c}$ & $\mathrm{c}$ & c & $\mathrm{c}$ & $\mathrm{c}$ & $\mathrm{c}$ & $\mathrm{c}$ & $\mathrm{C}$ & $\mathrm{c}$ \\
\hline Bat & $\mathrm{H}$ & $\mathrm{T}$ & $\mathrm{R}$ & $\mathrm{C}$ & C & $\mathrm{C}$ & $\mathrm{C}$ & C & $\mathrm{C}$ & $\mathrm{C}$ & $\mathrm{C}$ & C & $\mathrm{C}$ & $\mathrm{C}$ & C & C & C & C & C & c & C & C & $\mathrm{C}$ & $\mathrm{C}$ & $\mathrm{C}$ & $\mathrm{C}$ & C \\
\hline Elephant & $\mathrm{H}$ & s & $\mathrm{C}$ & $\mathrm{c}$ & $\mathrm{c}$ & $\mathrm{C}$ & $\mathrm{c}$ & $\mathrm{c}$ & $\mathrm{c}$ & $\mathrm{c}$ & $\mathrm{c}$ & $\mathrm{c}$ & $\mathrm{c}$ & c & c & $\mathrm{c}$ & c & $\mathrm{c}$ & $\mathrm{c}$ & $\mathrm{c}$ & c & $\mathrm{c}$ & $\mathrm{c}$ & $\mathrm{c}$ & $\mathrm{c}$ & C & $\mathrm{c}$ \\
\hline Chicken & - & - & - & C & c & C & c & c & $\mathrm{C}$ & $\mathrm{c}$ & $\mathrm{c}$ & c & c & c & c & G & C & c & $\mathrm{c}$ & c & A & C & $\mathrm{c}$ & $\mathrm{C}$ & c & c & C \\
\hline Zebrafish & - & - & $\mathrm{R}$ & $\mathrm{F}$ & C & C & C & C & C & C & C & A & 1 & C & C & $\mathrm{H}$ & C & C & $\mathrm{L}$ & $\mathrm{S}$ & $\mathrm{R}$ & V & $Y$ & C & C & $\mathrm{E}$ & $M$ \\
\hline
\end{tabular}

Figure 1. Protein disorder analyses and cysteine analyses for Nrf2 and Keap1. (A) Domain maps for Nrf2 and Keap1 show the location of all cysteine residues. Key oxidative stress-sensing cysteines in Keap1 are marked with an asterisk $\left(^{*}\right)$. (B) Disordered profile plots for Nrf2 and Keap1 predicting the location of intrinsically disordered regions within each protein as predicted by three 
independent algorithms (PrDOS, IUPred2A, and PONDR). The intrinsically disordered regions predicted by all three algorithms are highlighted in yellow. (C) The percentage of cysteine content is calculated for 15 species from human to zebrafish. (D) Protein sequence alignment for cysteine residues in Nrf2 and Keap1 across 15 species. All cysteines are highlighted in yellow. Sensor cysteines within Keap1 are marked with an asterisk $\left(^{*}\right)$.

\subsubsection{Oxidative stress and Nrf2 and Keap1 expression in yeast}

We previously established yeast as a useful tool to study Nrf2 interactions (Ngo et al., 2021; under review) and re-capitulated our data showing that that Nrf2 is toxic in yeast. Here, we again used growth assays to assess if Nrf2 expression in yeast is affected by the absence of oxidative stress genes. Human Nrf2 expressed in yeast caused 'toxicity', defined as an impaired growth phenotype on growth media compared to the empty vector control. Nrf2 was expressed in wild-type yeast and yeast strains deleted for an array of oxidative stress genes. Growth is quantified to the right as done previously [35] (Figure 2A). Only significant data is shown; for the complete list of deletion strains, refer to Table S2. Nrf2 toxicity was not significantly altered in these deletion strains. On the other hand, Keap1 expression in wild-type yeast was not toxic, but Keap1 toxicity was induced by the deletion of the antioxidant genes BTN1, SOD1, and TSA2 (Figure 2B). For the full panel of growth assays, refer to Figure S2 and Figure S3. For growth assay control plates, refer to Figure S4. Yeast cells expressing YFP-tagged wild-type Nrf2 or mutant variants of Nrf2, L30F and T80R, which have a reduced or impaired capacity to interact with Keap1 [13], were treated with $300 \mu \mathrm{M}$ hydrogen peroxide $\left(\mathrm{H}_{2} \mathrm{O}_{2}\right)$ for $3 \mathrm{~h}$ to elicit oxidative stress. A change in Nrf2 localization patterns was observed, as Nrf2-YFP was no longer diffusely spread throughout the yeast cytoplasm and nucleus but formed fluorescent foci (Figure $2 \mathrm{C})$. Moreover, when yeast cells expressing Keap1-YFP were treated with $300 \mu \mathrm{M} \mathrm{H}_{2} \mathrm{O}_{2}$, Keap1-YFP formed protein inclusions (Figure 2D). The optimal treatment dose and duration were determined by measuring cell viability in non-transfected HeLa cells to achieve a moderate, dose-dependent response to hydrogen peroxide treatment (Figure S5). These data indicate that increased oxidative stress exacerbates the toxicity of Nrf2 and induces inclusion formation of both Nrf2 and Keap1 in yeast. 
(A)

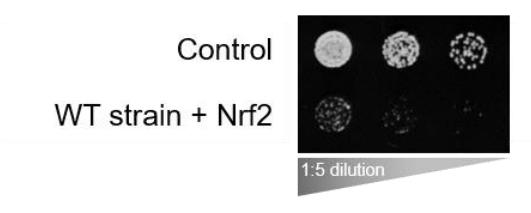

(B)

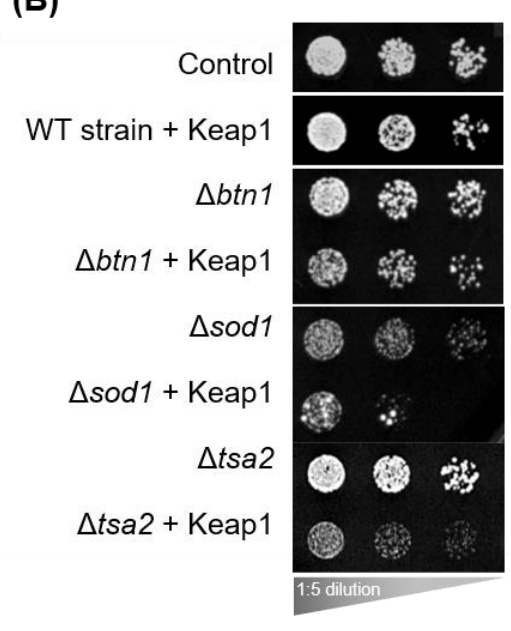

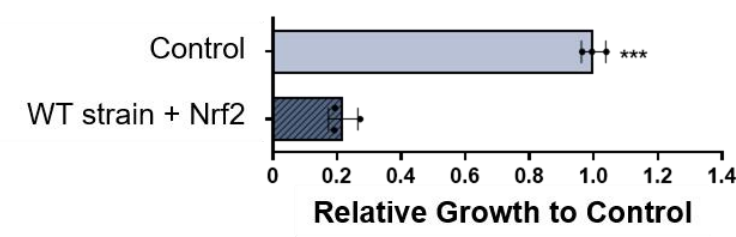

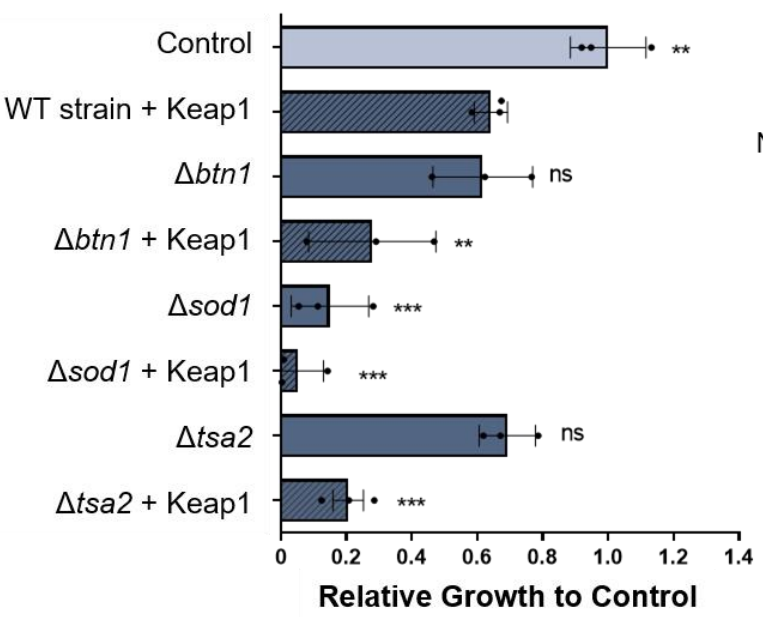

(C)

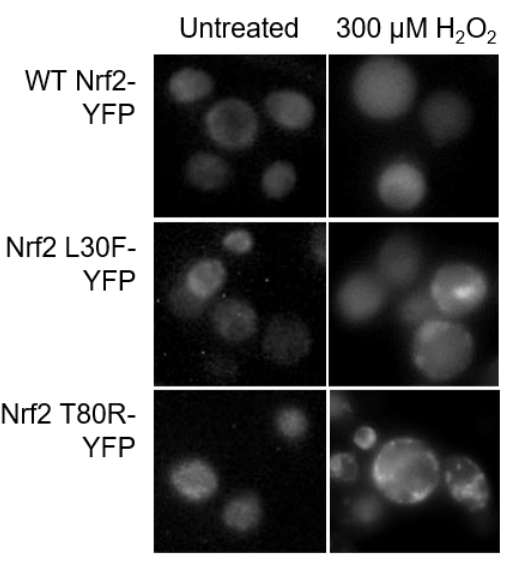

(D)

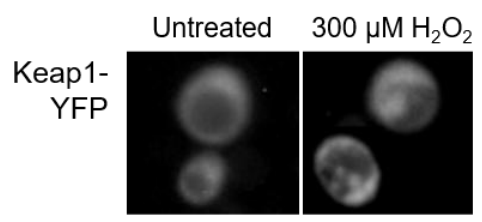

Figure 2. Oxidative stress and Nrf2 and Keap1 in yeast. Growth assays for (A) human Nrf2 and (B) human Keap1 transformed into yeast deletion strains for various oxidative stress genes grown on agar plates. Only significant data is shown. Growth is quantified to the right relative to the empty vector control. Means derived from three biological replicates were used during analysis. Means were analyzed using one-way ANOVA followed by Tukey's post hoc test. Data are expressed as mean \pm SD. $p<0.05$ was considered statistically significant; ${ }^{*}<<0.05,{ }^{* *} p<0.01,{ }^{* *} \mathrm{p}<0.001$. (C) Yeast expressing YFP-tagged Nrf2 and two Nrf2 mutants treated with 300 $\mu \mathrm{M} \mathrm{H}_{2} \mathrm{O}_{2}$. (D) Yeast expressing Keap1-YFP treated with $300 \mu \mathrm{M} \mathrm{H}_{2} \mathrm{O}_{2}$.

3.1.3. Nrf2 forms protein inclusions under oxidative stress conditions in HeLa cells Subsubsection

We next assessed whether Nrf2 also undergoes oxidative stress-induced inclusion formation in human cells. Figure $3 \mathrm{~A}$ documents the intramolecular localization of wildtype Nrf2 and two Nrf2 mutant variants with lowered affinity to Keap1, L30F and T80R, in HeLa cells. HeLa cells were transfected with wild-type or mutant green fluorescent protein (GFP)-tagged Nrf2 and treated with 100 or $300 \mu \mathrm{M} \mathrm{H}_{2} \mathrm{O}_{2}$ for $3 \mathrm{~h}$. The dose and duration were optimized in non-transfected HeLa cells as shown in Figure S5. As a negative control, cells were transfected with a pcDNA3.1-GFP empty vector for mammalian expression. Fluorescence microscopy revealed the formation of cytosolic and possibly nuclear protein inclusions of wild-type and mutant Nrf2 in both untreated and treated cells, at endogenous expression levels and even more so when Nrf2, L30F and T80R were overexpressed by transient transfection. In comparison, no stress-induced protein inclusions were observed for control HeLa cells transfected with GFP alone. The percentage of cells with inclusions increased in a hydrogen peroxide dose-dependent manner (Figure 3B). Nrf2 T80R shows a significantly higher percentage of cells with inclusions compared to wild-type Nrf2. Confocal microscopy was used to visualize wild-type Nrf2 inclusions at a higher resolution and Z-stacking reveals that Nrf2 inclusions are situated around the nucleus rather than within it (Figure 3C), thus, confirming these inclusions are cytosolic. 
(A)
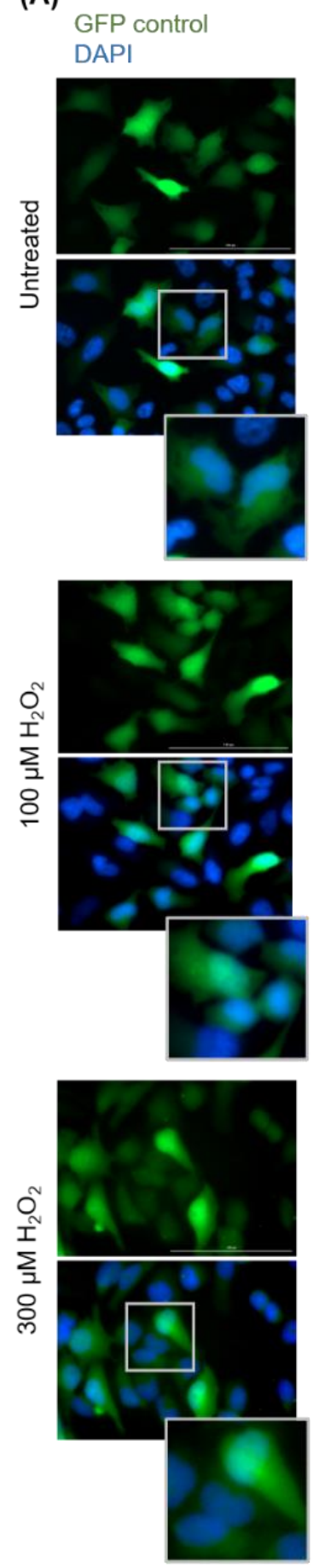

WT Nrf2-GFP

\section{DAPI}
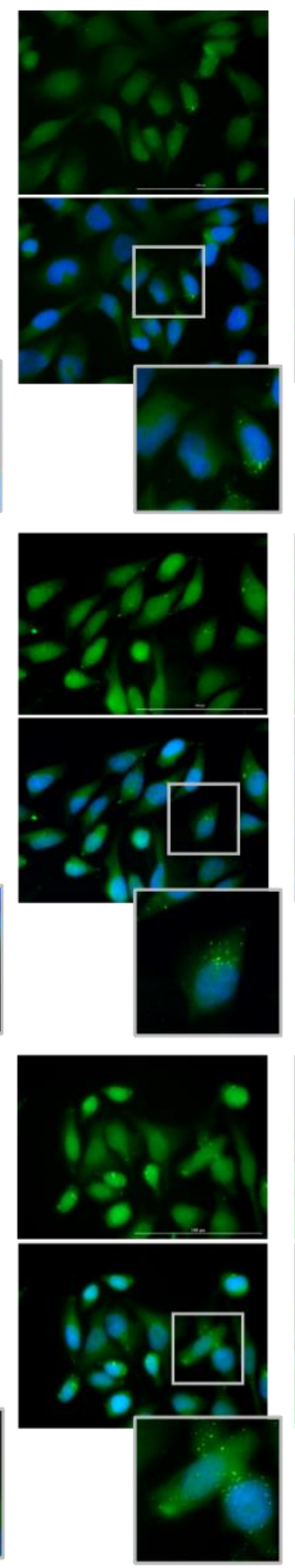

Nrf2 L30F-GFP DAPI
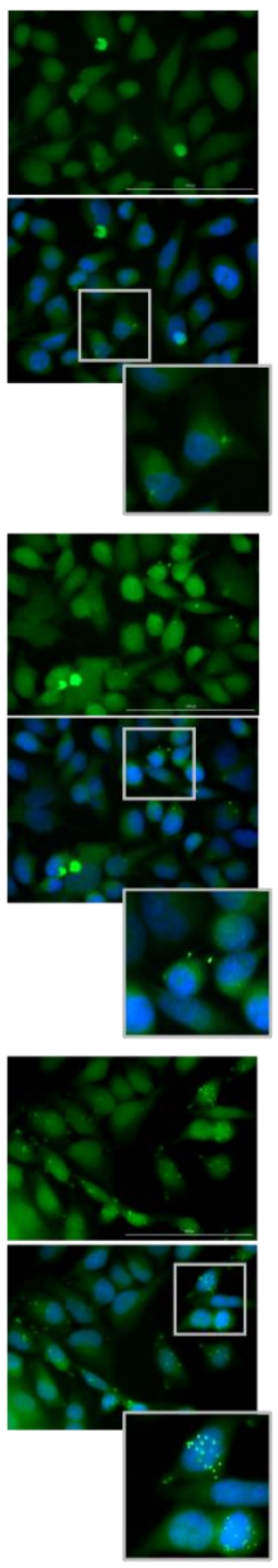

Nrf2 T80R-GFP DAPI
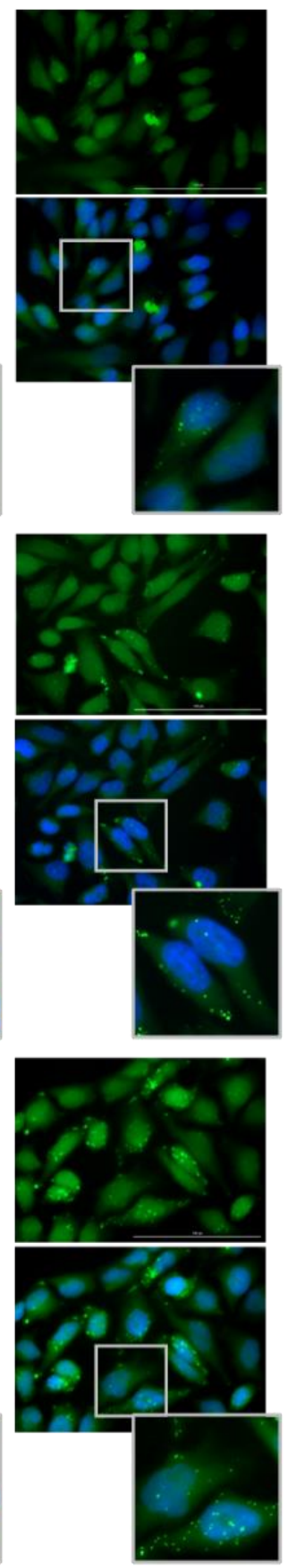

(B)

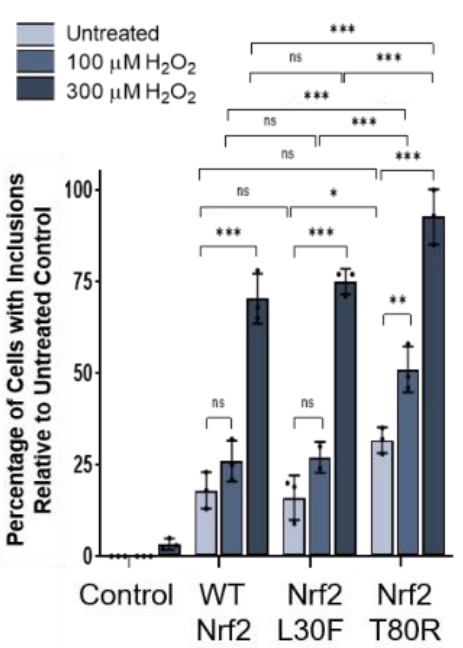

(C) WT Nrf2

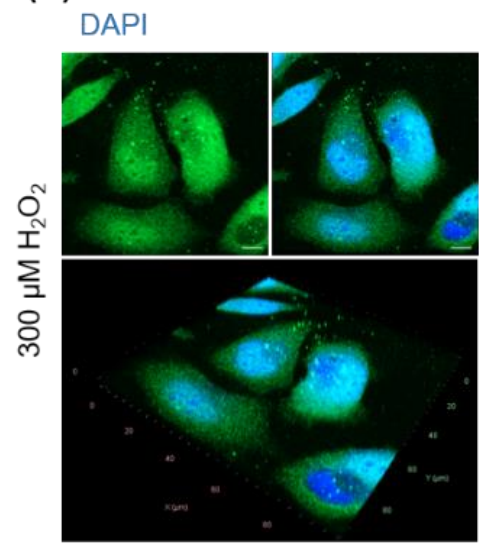

Figure 3. Nrf2 forms inclusions upon exposure to oxidative stress in vitro. (A) HeLa cells transfected with GFP-tagged Nrf2 and two Nrf2 mutants and treated with 100 or $300 \mu \mathrm{M} \mathrm{H}_{2} \mathrm{O}_{2}$ for $3 \mathrm{~h}$, visualized by fluorescence microscopy. As a negative control, cells were transfected with a pcDNA3.1-GFP empty vector for mammalian expression (note: the GFP channel for the control condition was underexposed to reduce overexposure of overly high-expression cells). (B) Quantification of Nrf2-expressing cells with inclusions following hydrogen peroxide treatment observed in (A). Means were analyzed using one-way ANOVA followed by Tukey's post hoc test. Data are expressed as mean \pm SD. $p<0.05$ was considered statistically significant; ${ }^{*} p<0.05,{ }^{* *} p<0.01,{ }^{* * *} p<0.001$. (C) Confocal microscopy with Z-stacking for wild-type Nrf2-expressing cells treated with hydrogen peroxide, visualized by immunofluorescence, and demonstrating that Nrf2 inclusions are cytosolic.

3.1.4. Nrf2 inclusion formation is oxidative stress-specific and prevented by certain antioxidants 
To determine whether oxidative-stress induced Nrf2 inclusion formation is an artifact of overexpression by transient transfection, un-transfected HeLa cells were treated with $300 \mu \mathrm{M} \mathrm{H}_{2} \mathrm{O}_{2}$ for $3 \mathrm{~h}$ and observed by immunofluorescence for endogenous Nrf2. The localization patterns for endogenous Nrf2 were similar to that of transfected Nrf2 with hydrogen peroxide treatment (Figure 4A), confirming that this observed effect is likely, not due to Nrf2 overexpression. To determine if Nrf2 inclusion formation is oxidative stress-specific, HeLa cells expressing Nrf2-GFP were treated with $50 \mu \mathrm{M}$ MG132, a proteasome inhibitor that elicits general protein misfolding stress. Compared to cells treated with $300 \mu \mathrm{M} \mathrm{H}_{2} \mathrm{O}_{2}$ which formed some Nrf2 inclusions, treatment with MG132 did not result in the formation of cytosolic Nrf2 inclusions (Figure 4B), indicating that inclusion formation was oxidative stress-specific.

Furthermore, we explored if treatment with antioxidants, such as N-acetylcysteine (NAC) and vitamin C (ascorbic acid), prevented Nrf2 inclusion formation. Transfected cells were pre-treated with $3 \mu \mathrm{M}$ NAC or $100 \mu \mathrm{M}$ vitamin C for $24 \mathrm{~h}$ and subsequently treated with $300 \mu \mathrm{M} \mathrm{H}_{2} \mathrm{O}_{2}$ for $3 \mathrm{~h}$. A reduction in $\mathrm{Nrf} 2$ inclusion formation was observed for pre-treatment with NAC but not vitamin C (Figure $4 \mathrm{C}$ ). Finally, we assessed the induction of Nrf2 (NFE2L2), Nrf2 target genes (HMOX1, NQO1) and thioredoxin (TXN) in HeLa cells following treatment with $300 \mu \mathrm{M} \mathrm{H} 2 \mathrm{O} 2$ for $3 \mathrm{~h}$ (Figure 4D). The Nrf2 target gene HMOX1 was induced with following hydrogen peroxide treatment but NQO1 was not, likely because the treatment duration of $3 \mathrm{~h}$ was insufficient for induction. Nonetheless, this suggests that Nrf2 is still functional as a transcription factor despite inclusion formation. 
(A)

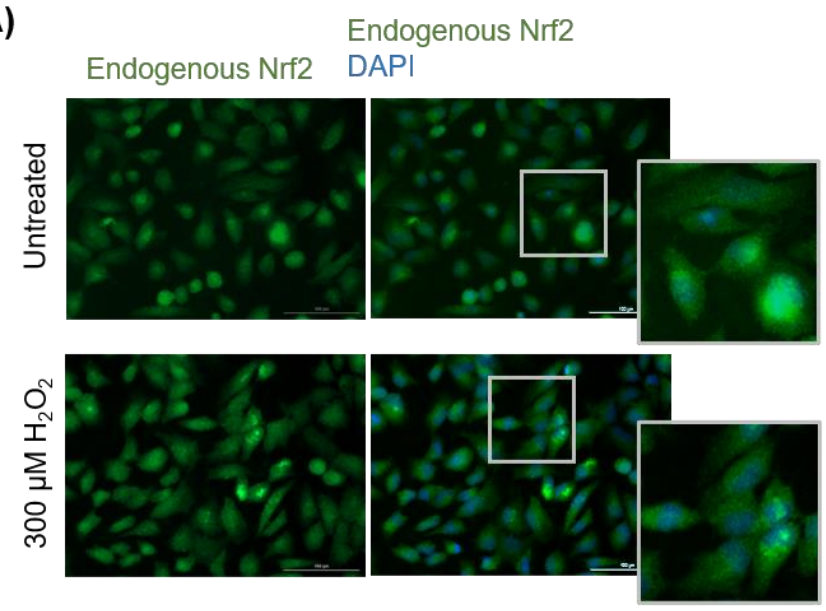

(C)

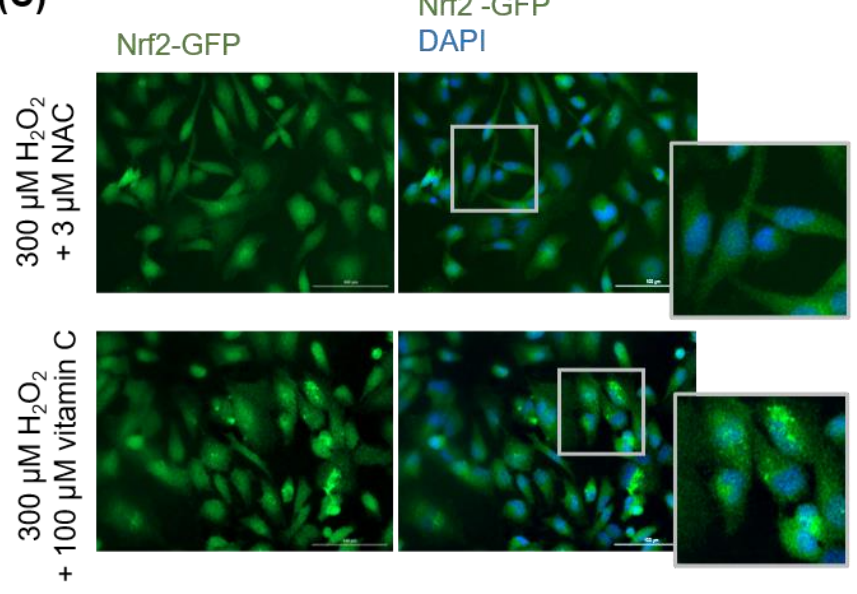

(B)

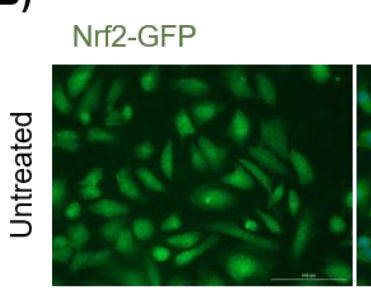

Nrf2-GFP

DAPI
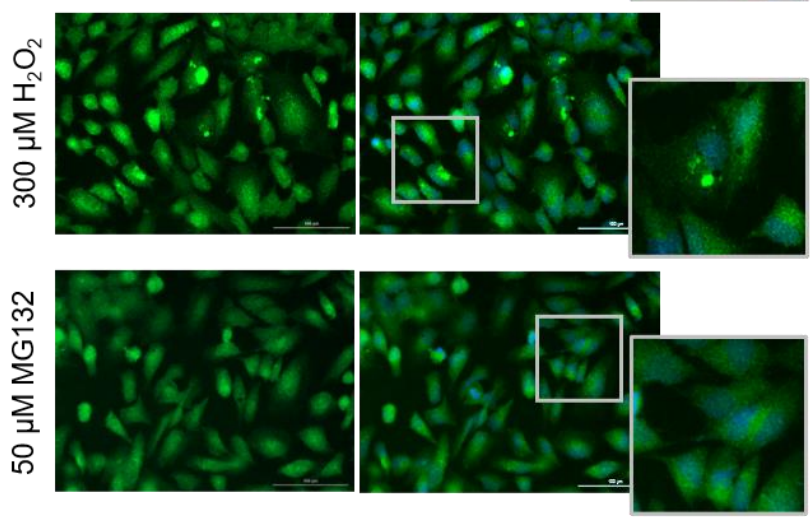

(D)

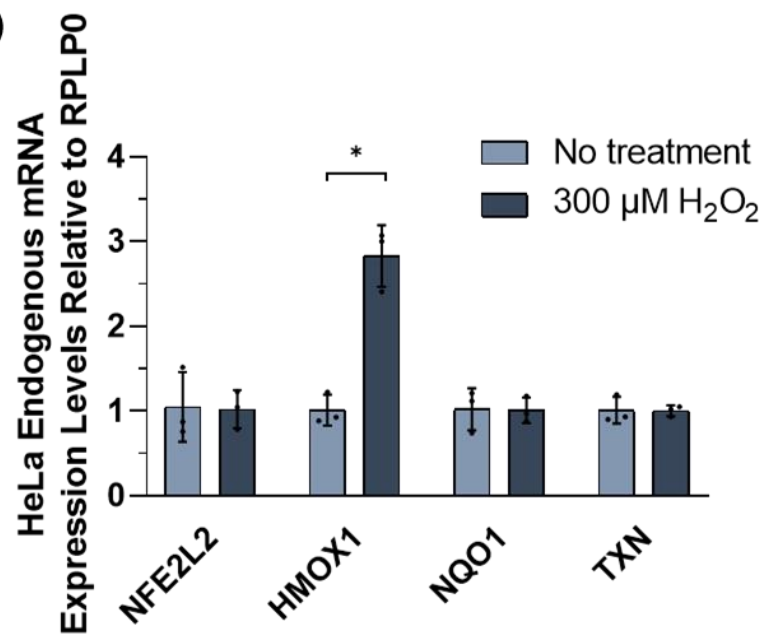

Figure 4. Further analyses of the oxidative stress-induced protein misfolding of Nrf2.

(A) Endogenous Nrf2 in untransfected HeLa cells treated with $300 \mu \mathrm{M} \mathrm{H}_{2} \mathrm{O}_{2}$ for $3 \mathrm{~h}$, visualized by immunofluorescence microscopy. (B) Transfected Nrf2-GFP in HeLa cells treated with $50 \mu \mathrm{M}$ MG132 for 6 h, visualized by fluorescence microscopy. (C) Transfected Keap1-GFP in HeLa cells pretreated with $3 \mu \mathrm{M} \mathrm{NAC}$ and or $100 \mu \mathrm{M}$ vitamin $\mathrm{C}$ for $24 \mathrm{~h}$ followed by treatment with $300 \mu \mathrm{M} \mathrm{H}_{2} \mathrm{O}_{2}$ for $3 \mathrm{~h}$, visualized by fluorescence microscopy. (D) Relative mRNA levels of Nrf2 (NFE2L2), Nrf2 target genes (HMOX1, NQO1), and thioredoxin (TXN) were evaluated by RT-qPCR following treatment of HeLa cells with $300 \mu \mathrm{M} \mathrm{H}_{2} \mathrm{O}_{2}$ for $3 \mathrm{~h}$. Data were normalized to RPLP0 transcript levels. Means derived from three biological replicates were used during analysis.

\subsection{Analysis of Keap1}

\subsubsection{Keap1 forms protein inclusions under oxidative stress conditions in HeLa cells}

HeLa cells were transfected with GFP-tagged Keap1 and treated with 100 or $300 \mu \mathrm{M}$ $\mathrm{H}_{2} \mathrm{O}_{2}$ for 3 hours. Fluorescence microscopy revealed the formation of cytosolic and possibly nuclear protein inclusions in both untreated and treated cells (Figure 5A). with the percentage of cells with protein inclusions quantified to the right. Quantification of the percentage of cells containing inclusions revealed that the oxidative stress-induced Keap1 inclusions formed in a hydrogen peroxide dose-dependent manner (Figure 5B). Confocal microscopy was used to visualize these inclusions at a higher resolution (Figure 5C) and Z-stacking revealed that Keap1 inclusions were situated around the nucleus rather than within it (Figure 5D), thus, confirming these inclusions were cytosolic. 
(A)

Keap1-GFP DAPI

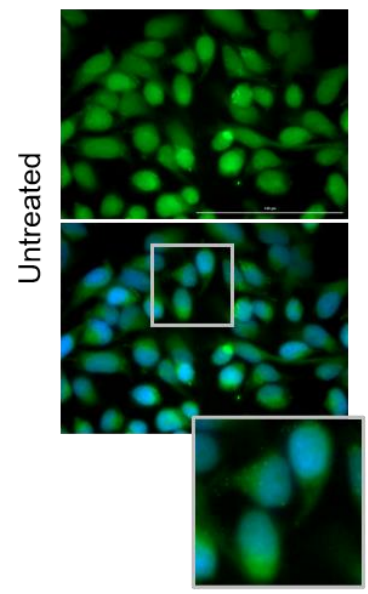

Keap1-GFP DAPI

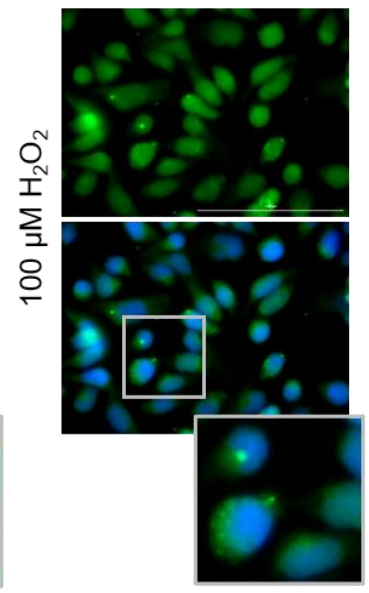

Keap1-GFP DAPI

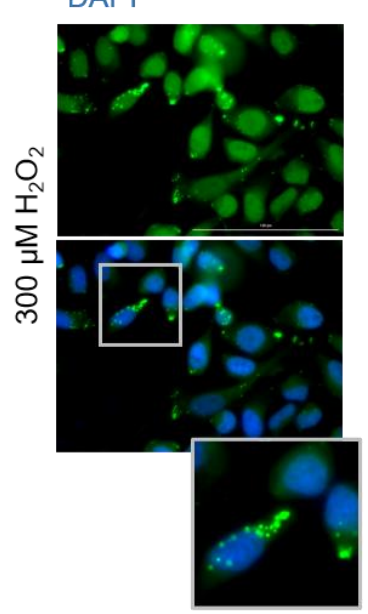

(B)

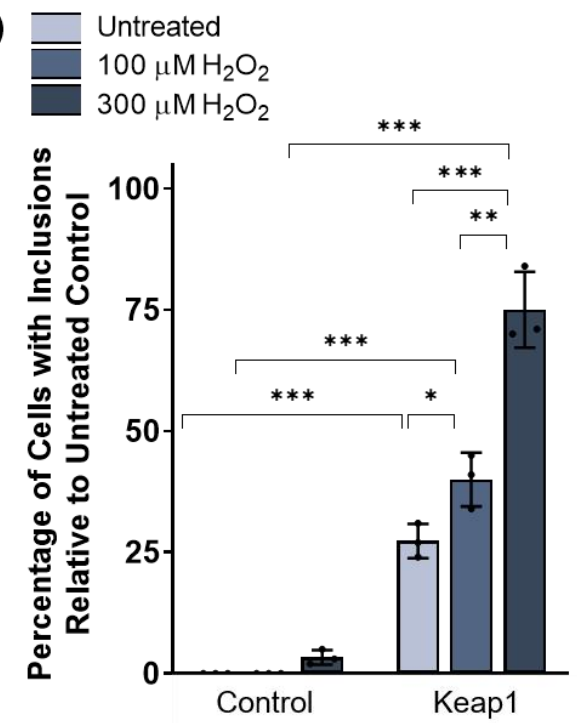

(D) Keap1 DAPI $\quad 300 \mu \mathrm{M} \mathrm{H}_{2} \mathrm{O}_{2}$
(C) Keap1

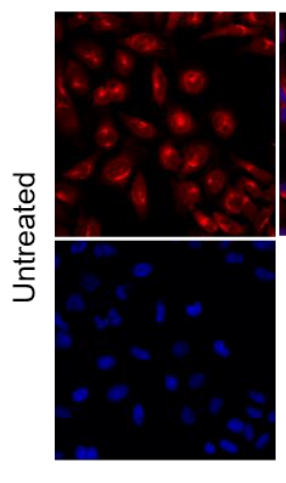

Keap1

DAPI

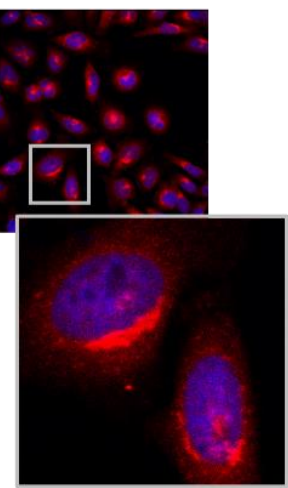

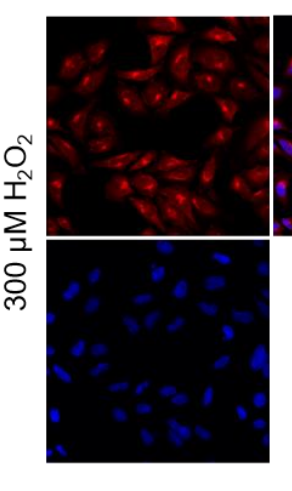
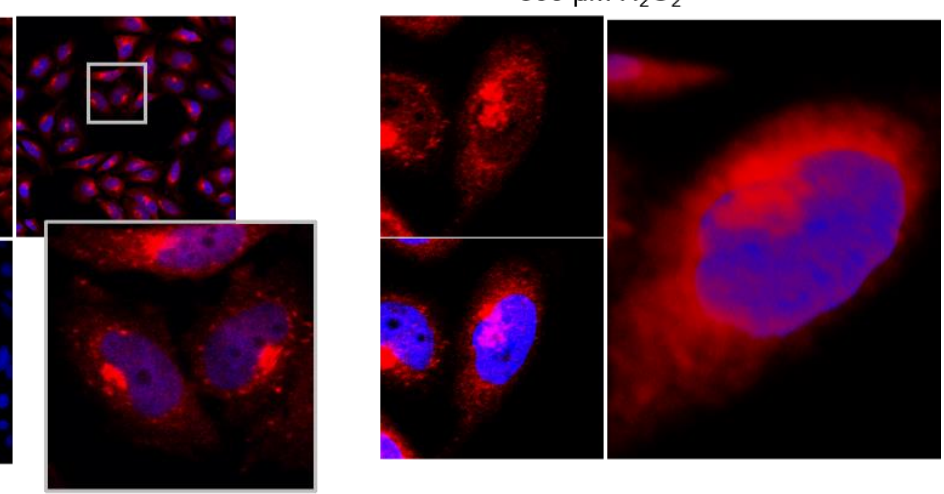

Figure 5. Keap1 forms inclusions upon exposure to oxidative stress in vitro. (A) HeLa cells transfected with Keap1-GFP and treated with 100 or $300 \mu \mathrm{M} \mathrm{H}_{2} \mathrm{O}_{2}$ for $3 \mathrm{~h}$, visualized by fluorescence microscopy. (B) Quantification of Keap1-expressing cells with inclusions following hydrogen peroxide treatment in (A). Means derived from three biological replicates were used during analysis. Means were analyzed using one-way ANOVA followed by Tukey's post hoc test. Data are expressed as mean \pm SD. $\mathrm{p}<0.05$ was considered statistically significant; ${ }^{*} \mathrm{p}<0.05,{ }^{* *} \mathrm{p}<0.01,{ }^{* * *} \mathrm{p}<0.001$. (C) Confocal microscopy for Keap1-expressing cells treated with $300 \mu \mathrm{M} \mathrm{H}_{2} \mathrm{O}_{2}$ for $3 \mathrm{~h}$, visualized by immunofluorescence. (D) Confocal microscopy with Z-stacking for Keap1-expressing cells treated with hydrogen peroxide, visualized by immunofluorescence, and demonstrating that Keap1 inclusions are cytosolic.

3.2.2. Keap1 inclusion formation is oxidative stress-specific and is not prevented by antioxidants

Again, to determine whether oxidative stress-induced Keap1 inclusion formation was an artifact of overexpression by transient transfection, un-transfected HeLa cells were treated with $300 \mu \mathrm{M} \mathrm{H}_{2} \mathrm{O}_{2}$ for $3 \mathrm{~h}$ and observed using immunofluorescence microscopy for endogenous Keap1. Endogenous Keap1 also formed inclusions upon treatment with hydrogen peroxide (Figure 6A), confirming that this observed effect is not due to Keap1 overexpression. To determine if Keap1 inclusion formation is oxidative stress-specific, HeLa cells expressing Keap1-GFP were treated with $50 \mu \mathrm{M}$ MG132 to elicit general protein misfolding stress. Compared to cells treated with $300 \mu \mathrm{M} \mathrm{H}_{2} \mathrm{O}_{2}$ which formed Keap1 inclusions, treatment with MG132 did not result in the formation of inclusions (Figure 6B).

Finally, we determined if NAC and vitamin C could prevent Keap1 inclusion formation upon oxidative stress. Transfected cells were pre-treated with $3 \mu \mathrm{M}$ NAC or 100 $\mu \mathrm{M}$ vitamin $\mathrm{C}$ for $24 \mathrm{~h}$ and subsequently treated with $300 \mu \mathrm{M} \mathrm{H}_{2} \mathrm{O}_{2}$ for $3 \mathrm{~h}$; however, no significant reduction in Keap1 inclusions was observed (Figure 6C), which contrasts our results with Nrf2 where NAC can indeed reverse inclusion formation. 

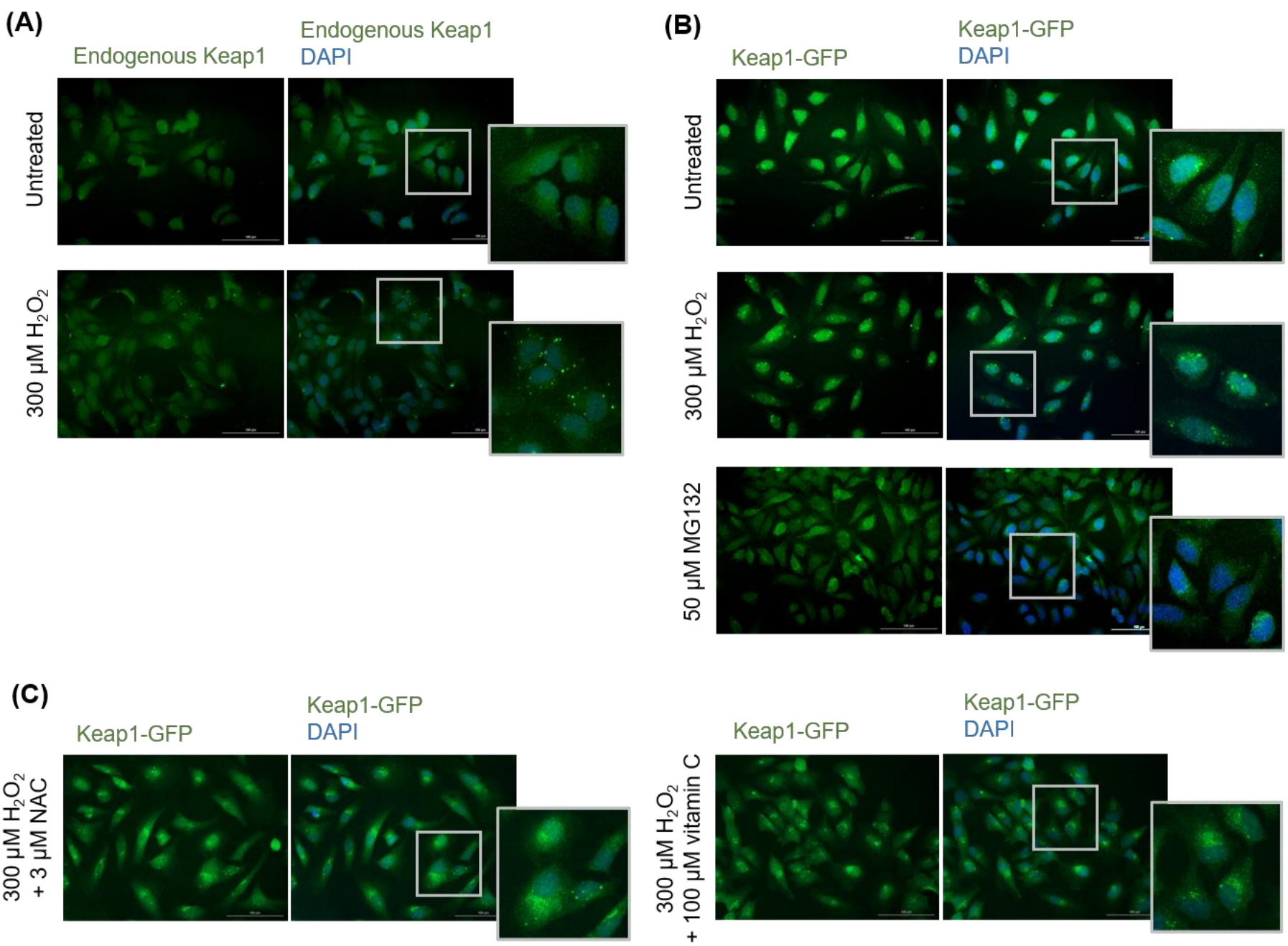

Figure 6. Further analyses of Keap1 oxidative stress-induced protein misfolding. (A) Endogenous Keap1 in un-transfected HeLa cells treated with $300 \mu \mathrm{M} \mathrm{H}_{2} \mathrm{O}_{2}$ for $3 \mathrm{~h}$, visualized by immunofluorescence microscopy. (B) Transfected Keap1-GFP in HeLa cells treated with $50 \mu \mathrm{M}$ MG132 for $6 \mathrm{~h}$, visualized by fluorescence microscopy. (C) Transfected Keap1-GFP in HeLa cells pretreated with $3 \mu \mathrm{M}$ NAC or $100 \mu \mathrm{M}$ vitamin $\mathrm{C}$ for $24 \mathrm{~h}$ followed by treatment with $300 \mu \mathrm{M} \mathrm{H}_{2} \mathrm{O}_{2}$ for 3 h, visualized by fluorescence microscopy.

\subsubsection{Oxidative stress-induced Keap1 inclusion formation in breast cancer cell lines}

To ensure that Keap1 stress-induced inclusion formation was not a HeLa or HEK293 cell line-specific phenomenon, we treated two human breast cancer cell lines, 21MT-1 and SKBR3, with $300 \mu \mathrm{M} \mathrm{H}_{2} \mathrm{O}_{2}$ for $3 \mathrm{~h}$ and performed immunofluorescence microscopy for endogenous Keap1. Upon oxidative stress, both cell lines showed Keap1 inclusions (Figure $7 \mathrm{~A}$ and Figure $7 \mathrm{~B}$ ), quantified to be statistically significant from the untreated control (Figure 7C). 


\section{(A)}

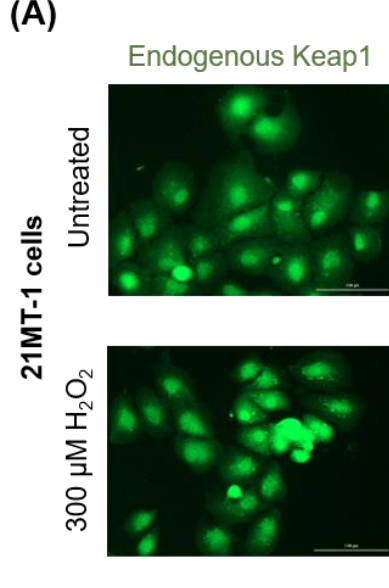

(B)

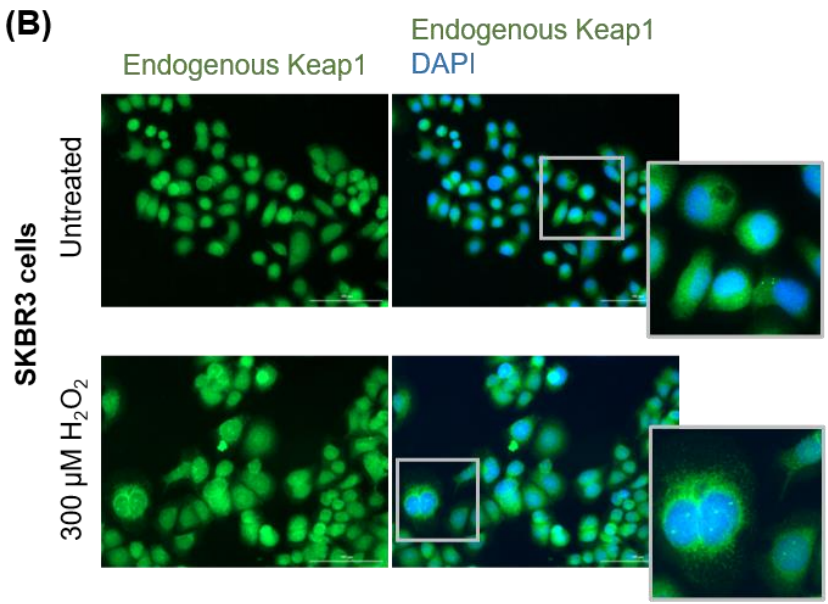

Endogenous Keap1

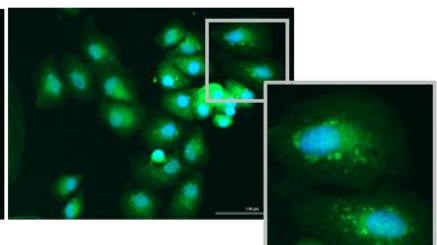

Endogenous Keap1
(C)

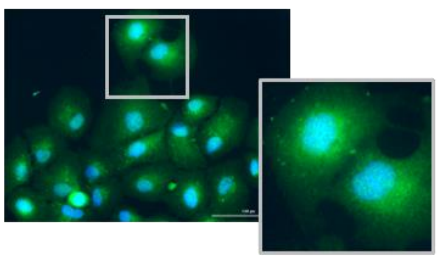

\section{.}

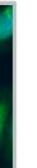

Figure 7. Keap1 forms oxidative stress-induced inclusions in breast cancer cell lines. (A-B) Endogenous Keap1 expression in two breast cancer cell lines, 21MT-1 (A) and SKBR3 (B), treated with $300 \mu \mathrm{M} \mathrm{H} 2 \mathrm{O} \neg 2$ for $3 \mathrm{~h}$, visualized by immunofluorescence microscopy. (C) Quantification of Keap1 inclusions following hydrogen peroxide treatment in (A, B). Means derived from three biological replicates were used during analysis. Means were analyzed using one-way ANOVA followed by Tukey's post hoc test. Data are expressed as mean \pm SD. $p<0.05$ was considered statistically significant; ${ }^{*} \mathrm{p}<0.05,{ }^{* *} \mathrm{p}<0.01,{ }^{* * *} \mathrm{p}<0.001$.

\subsection{Analysis of Nrf2 and Keap1 purified protein}

3.3.1. Purified proteins for Nrf2 and Keap1 form inclusions upon exposure to oxidative stress

Purified proteins were used to biochemically assess Nrf2 and Keap1 misfolding and inclusion formation (or aggregation) using two methods: (1) traditional SDS-PAGE with Coomassie blue gel staining, and (2) semi-denaturing detergent agarose gel electrophoresis (SDD-AGE) with western bot analysis. As shown in Figure 8A, purified Nrf2 was incubated with $600 \mu \mathrm{M} \mathrm{H}_{2} \mathrm{O}_{2}$ in the absence and presence of reducing agent $\beta$-mercaptoethanol $(\beta M E)$. Upon treatment with hydrogen peroxide, Nrf2 formed a dense high molecular weight smear, indicating the formation of aggregated, higher molecular weight species. With the addition of $5 \%$ of the reducing reagent $\beta \mathrm{ME}$, this structure collapsed, indicating that this high molecular weight protein species is, to some degree, dependent upon disulfide bonds. Furthermore, analysis of purified Neh5 domain of Nrf2, which harbours one of the six cysteines in the protein, revealed the formation of an aggregated, higher molecular weight species with hydrogen peroxide treatment that also collapses upon $\beta \mathrm{ME}$ treatment (Figure 8B).

Similarly, purified Keap1 protein was treated with $600 \mu \mathrm{M} \mathrm{H}_{2} \mathrm{O}_{2}$ in the presence and absence of $\beta \mathrm{ME}$. Upon treatment with hydrogen peroxide, Keap1 formed a dense high molecular weight smear, indicating the formation of an aggregated, higher molecular weight species. With the addition of $5 \% \beta \mathrm{ME}$, this structure collapsed (Figure $8 \mathrm{C}$ ), indicating that this high molecular weight protein species was also, at least in part, dependent 
on the formation of disulfide bonds. Purified protein for Keap1's Kelch domain was then used to determine if this well-folded domain alone, which contains only eight cysteine residues, would misfold under oxidative stress conditions. Unlike full-length Keap1, treatment of the Kelch domain with $600 \mu \mathrm{M} \mathrm{H}_{2} \mathrm{O}_{2}$ did not produce a high molecular weight species (Figure 8D).

Finally, fractionation assays were used to determine the soluble and aggregated fractions for purified Nrf2 and Keap1 (Figure 8E). The total purified protein sample was centrifuged and divided into the soluble supernatant fraction and the aggregated pellet fraction and resolved using traditional SDS-PAGE with Coomassie blue gel staining. Both Nrf2 and Keap1 contained soluble and aggregated protein fractions in the supernatant and pellet, respectively.

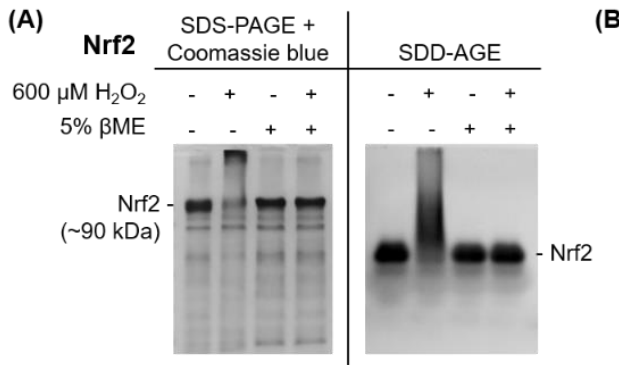

(B)

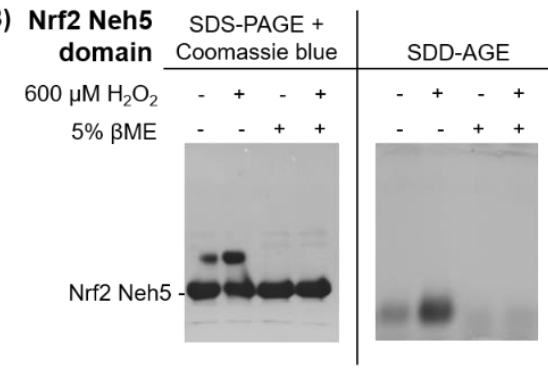

(D)
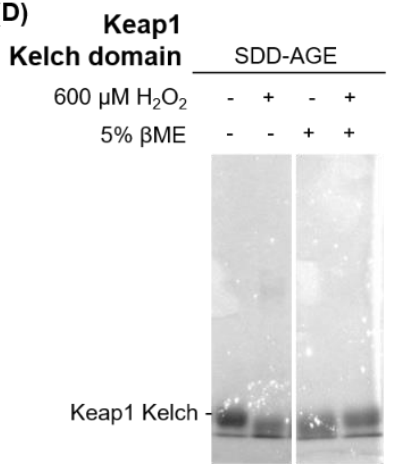

(E)

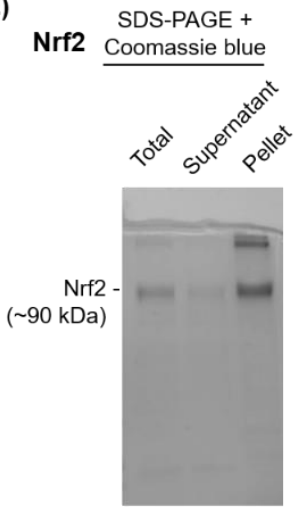

(C)
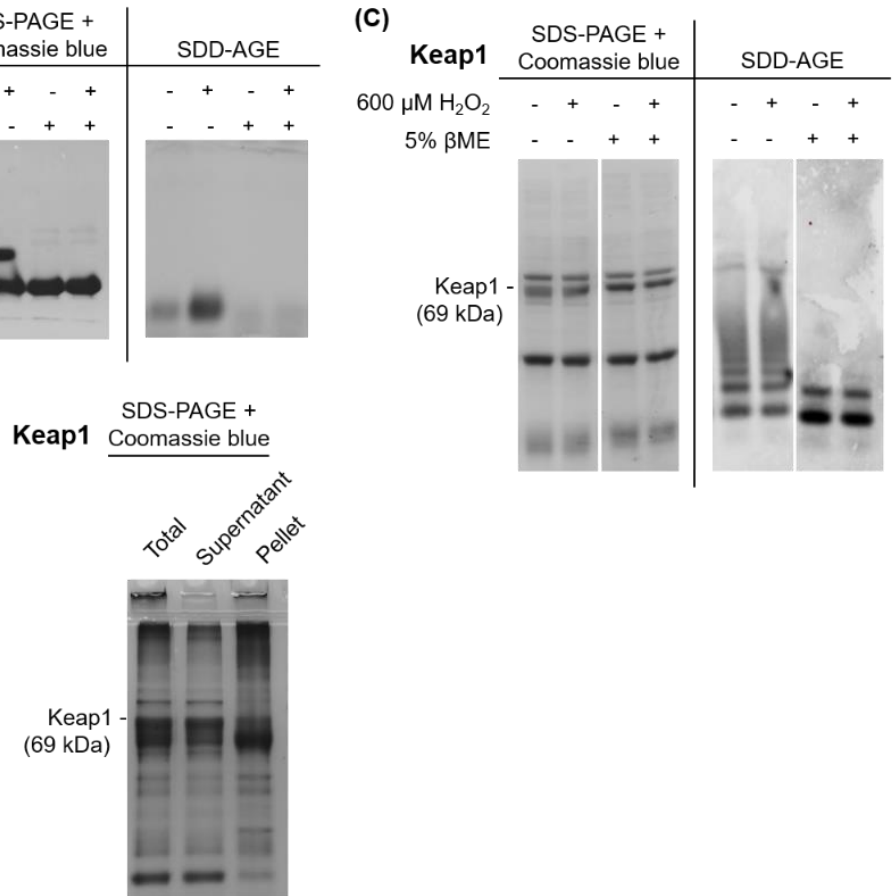

$5 \%$ BME

Keap1 -

$(69 \mathrm{kDa})$

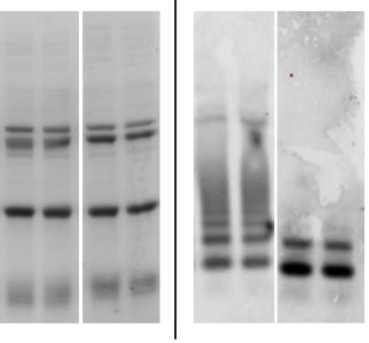

Figure 8. Nrf2 and Keap1 purified proteins aggregate upon exposure to oxidative stress in vitro. Purified protein treated with 600 $\mu \mathrm{M} \mathrm{H}_{2} \mathrm{O}_{2} \pm \beta \mathrm{ME}$ for 30 mins for (A) Nrf2, (B) Nrf2's Neh5 domain, (C) Keap1, and (D) Keap1's Kelch domain. (E) Fractionation assay for purified Nrf2 and Keap1 protein. All purified proteins are resolved using SDS-PAGE with Coomassie Blue gel staining and/or SDD-AGE, as indicated.4. Discussion

In this work, we demonstrate that two key proteins of the antioxidant pathway, Nrf2 and Keap1, form intracellular inclusions upon exposure to high levels of oxidative stress. We believe that at least in part, the aberrant formation of disulfide bonds causes the misfolding and inclusion formation of both proteins. Nrf2's intrinsically disordered nature may also contribute to its propensity to misfold and form inclusions

We observe that in both yeast and mammalian cells (HeLa and breast cancer cell lines) the treatment of cells expressing Nrf2 with hydrogen peroxide results in the formation of protein inclusions in a dose-dependent manner. Interestingly, protein inclusion formation was exacerbated for the Nrf2 mutant variants, L30F and T80R, where Keap1binding to the Neh2 domain in Nrf2 at the low- and high-affinity motifs, respectively, is impaired, resulting in loss of Keap1-mediated degradation [13, 14]. Oxidatively damaged Nrf2 accumulates in the cytosol as inclusions, most notably for the Nrf2 T80R mutant which escapes all Keap1-mediated degradation. This could explain the significantly high levels of Nrf2 T08R inclusions. On the other hand, Nrf2 L30F demonstrates impaired binding at the low-affinity motif but may still retain some Nrf2-binding via the high-affinity motif. Nonetheless, ubiquitination of Nrf2 is unlikely without the intact low-affinity binding site [15]. Future work will further investigate the functional consequences of these 
mutants and how impaired Keap1-dependent Nrf2 regulation affects oxidative stress-induced inclusion formation.

Numerous domains of Nrf2 have previously been characterized as intrinsically disordered $[14,16]$ and our data support this. While this structural flexibility could allow Nrf2 to bind to a large number of different proteins [39], it may also render Nrf2 susceptible to aberrant protein misfolding. Intrinsically disordered proteins tend to misfold under certain conditions [40] which appears to also be the case for Nrf2 during high levels of oxidative stress. This misfolding and inclusion formation of Nrf2 could be an adaptive mechanism of Nrf2 regulation, i.e., the 'functional misfolding' of IDPs [40], which allows Nrf2 to escape degradation, resulting in strong constitutive ARE activation. Alternatively, this could be a maladaptive mechanism, i.e., misfolded Nrf2 cannot activate ARE-containing genes in response to oxidative stress. To determine this, future work must assess the functional outcome of this stress-induced Nrf2 misfolding and its consequences on Nrf2 regulation and activity.

Moreover, wild-type Nrf2 contains only six cysteine residues but our in vitro experiments document that at least some of the cysteines in Nrf2 are oxidized and are central to the formation of high molecular weight protein species. It is plausible that oxidation-induced inclusion formation impairs the transcriptional activity of Nrf2, as $\mathrm{He}$ et al. have found that some cysteine residues of Nrf2 play important roles in oxidant sensing, Keap1dependent ubiquitination and degradation of Nrf2, and in the transcriptional activation of ARE-containing Nrf2 target genes [41]. In contrast, Keap1 is not intrinsically disordered and its misfolding may be mostly dependent on the presence of cysteine residues that are highly susceptible to oxidation. Keap1 contains 27 cysteines, 24 of which were found to be highly or completely conserved. Interestingly, except for the chicken and zebrafish, all key sensor cysteines within Keap1 [7-9, 36] were mostly completely conserved, which demonstrates their importance for Keap1 function. Cysteines are one of the least abundant amino acids in mammals, comprising an average of $2.3 \%$ for the human proteome [38]. Yet we find that human Keap1 contains $4.33 \%$ cysteine content, almost double the average for the human proteome [38]. This high content of cysteine residues in Keap1 may render it more susceptible to oxidation, for example by aberrant disulfide bond formation. Fourquet et al. have shown that hydrogen peroxide can oxidize Keap1 [42]. We furthermore show that that cysteine oxidation Keap1 may contribute to the formation of inclusions and high molecular weight protein species upon treatment with hydrogen peroxide in yeast, mammalian cells, and as purified proteins, and that treatment of Keap1 (and Nrf2) purified proteins with a reducing agent to break disulfide bonds results in the solubilization of the aggregated species. Of note, our in vitro data argue that the cysteines within the stably folded Kelch domain of Keap1 are not susceptible to oxidation and misfolding for the Kelch domain alone, which is consistent with the notion that not all cysteine residues within Keap1 are equally reactive. Future work must determine which cysteines in Keap1 are most susceptible to oxidation and inclusion formation (e.g., by cysteine mutation analyses), and if the full-length protein is required for this misfolding to occur.

Under normal conditions, Keap1 is constantly shuttling between the cytosol and the nucleus via importin $\alpha 7$ (also known as karyopherin $\alpha 6$, KPNA6), and the nuclear import of Keap1 represses the Nrf2 antioxidant response [43]; however, our results show that misfolded Keap1 inclusions are cytosolic. Misfolded Keap1 may render the protein inactive, impairing Keap1-mediated degradation of both cytosolic and nuclear Nrf2. We speculate that Keap1, upon forming aberrant intra- and/or inter-molecular disulfide bonds, cannot bind to Nrf2 to target it for degradation, thus allowing free Nrf2 to activate the antioxidant response. It is plausible that this Keap1 inactivation is irreversible and that even newly synthesized Keap1 cannot escape oxidation-based inactivation under high levels of oxidative stress, thus causing a long-lasting constitutive activation of Nrf2. This mechanism may be predominant in cancer, where ROS levels can be high due to increased metabolic activity and genetic instability [44]. Importantly, under the oxidative stress conditions that we explored (i.e., $300 \mu \mathrm{M} \mathrm{H}_{2} \mathrm{O}_{2}$ for $3 \mathrm{~h}$ ), misfolded $\mathrm{Nrf2}$ remains functional and was still able to induce the Nrf2 target gene HMOX1; however, Nrf2 target gene 
NQO1 was not induced, likely due to an insufficient treatment duration for this particular gene.

It is important to mention the work by Taguchi et al. which proposes that oxidative stress causes Keap1 misfolding and its sequestration into inclusion bodies that are removed by p62/SQSTM1 [45]. This is a mechanism that is not mutually exclusive to the one proposed in this study; however, it is also important to note that the inclusion bodies observed by Taguchi et al. seem to differ in morphology compared to the inclusions observed in this study and that the end product of the p62-Keap1 interaction is Keap1 degradation by autophagy, which is not observed here. In addition, purified Keap1 protein misfolds and forms inclusions upon oxidative stress treatment in a purified protein 'test tube' scenario where p62 is absent.

Taken together, we employed yeast models, cultured mammalian cells, and purified proteins to assess the oxidative stress-induced inclusion formation of Nrf2 and Keap1. We argue that the intrinsically disordered nature of Nrf2 exposes its cysteine residues to ROS and thus makes it more prone to misfolding, while the more structured Keap1's unusually high content of cysteine residues makes the protein more susceptible to misfolding by aberrant disulfide bond formation. Whether this mechanism is involved in physiologic Nrf2 regulation during stress or a pathological mechanism in hostile cell stress conditions remains to be explored. Our work provides new and preliminary insight into previously unexplored aspects of Nrf2 regulation by oxidation-dependent protein inclusion formation. Future work will seek to explore the functional outcome of this oxidation event in normal cells and cancer cells.

Supplementary Materials: The following are available online at www.mdpi.com/xxx/, Figure S1: Individual disordered profile plots for PrDOS, IUPred2U, and PONDR, Figure S2: Full panel of growth assays for Nrf2 expression in yeast oxidative stress deletion strains, Figure S3: Full panel of growth assays for Keap1 expression in yeast oxidative stress deletion strains, Figure S4: Growth assay control plates for all yeast oxidative stress deletion studies, Figure S5: Optimization of hydrogen peroxide treatment concentration and duration, Figure S6: Full blots for all Coomassie blue and SDD-AGE experiments in Figure 8, Table S1: The 15 species observed in cysteine analysis studies, Table S2: Yeast oxidative stress gene deletion strains used in this study.

Author Contributions: Conceptualization, V.N. and M.L.D.; methodology, V.N., and N.C.K., Z.S., and A.B. assisted with protein purification; formal analysis, V.N. and M.L.D.; resources, M.L.D.; writing-original draft preparation, V.N. and M.L.D.; writing-review and editing, V.N., A.B., W.Y.C. and M.L.D.; supervision, M.L.D.; funding acquisition, V.N. and M.L.D. All authors have read and agreed to the published version of the manuscript.

Funding: This research was funded by a Lawson Research Grant to M.L.D. V.N. was the recipient of a Queen Elizabeth II Graduate Scholarship in Science and Technology, a Breast Cancer Society of Canada Translational Research Unit studentship, and a Dr. Frederick Winnett Luney Graduate Scholarship in Pathology and Laboratory Medicine.

Acknowledgments: We thank Dr. Asit Rai and Zheng Song for his technical assistance with confocal microscopy and protein purification, respectively. We thank Dr. Christopher Howlett and Dr. Patrick Lajoie for their advice and helpful discussions throughout the course of this work.

Conflicts of Interest: The authors declare no conflict of interest.

\section{References}

1. Moi, P., et al., Isolation of NF-E2-related factor 2 (Nrf2), a NF-E2-like basic leucine zipper transcriptional activator that binds to the tandem NF-E2/AP1 repeat of the beta-globin locus control region. Proceedings of the National Academy of Sciences, 1994. 91(21): p. 9926-9930.

2. Itoh, K., et al., Keap1 represses nuclear activation of antioxidant responsive elements by Nrf2 through binding to the aminoterminal Neh2 domain. Genes Dev, 1999. 13(1): p. 76-86. 
3. McMahon, M., et al., Keap1-dependent proteasomal degradation of transcription factor Nrf2 contributes to the negative regulation of antioxidant response element-driven gene expression. J Biol Chem, 2003. 278(24): p. 21592-600.

4. Itoh, K., et al., Keap1 regulates both cytoplasmic-nuclear shuttling and degradation of Nrf2 in response to electrophiles. Genes Cells, 2003. 8(4): p. 379-91.

5. Nguyen, T., et al., Increased protein stability as a mechanism that enhances Nrf2-mediated transcriptional activation of the antioxidant response element. Degradation of Nrf2 by the $26 \mathrm{~S}$ proteasome. J Biol Chem, 2003. 278(7): p. 4536-41.

6. Kobayashi, A., et al., Oxidative and electrophilic stresses activate Nrf2 through inhibition of ubiquitination activity of Keap1. Mol Cell Biol, 2006. 26(1): p. 221-9.

7. Zhang, D.D. and M. Hannink, Distinct cysteine residues in Keap1 are required for Keap1-dependent ubiquitination of Nrf2 and for stabilization of Nrf2 by chemopreventive agents and oxidative stress. Mol Cell Biol, 2003. 23(22): p. 8137-51.

8. Dinkova-Kostova, A.T., et al., Direct evidence that sulfhydryl groups of Keap1 are the sensors regulating induction of phase 2 enzymes that protect against carcinogens and oxidants. Proceedings of the National Academy of Sciences, 2002. 99(18): p. 11908.

9. Wakabayashi, N., et al., Protection against electrophile and oxidant stress by induction of the phase 2 response: fate of cysteines of the Keap1 sensor modified by inducers. Proc Natl Acad Sci U S A, 2004. 101(7): p. 2040-5.

10. Yamamoto, M., T.W. Kensler, and H. Motohashi, The KEAP1-NRF2 System: a Thiol-Based Sensor-Effector Apparatus for Maintaining Redox Homeostasis. Physiol Rev, 2018. 98(3): p. 1169-1203.

11. Baird, L. and M. Yamamoto, The Molecular Mechanisms Regulating the KEAP1-NRF2 Pathway. Mol Cell Biol, 2020. 40(13).

12. Padmanabhan, B., et al., Structural basis for defects of Keap1 activity provoked by its point mutations in lung cancer. Mol Cell, 2006. 21(5): p. 689-700.

13. Shibata, T., et al., Cancer related mutations in NRF2 impair its recognition by Keap1-Cul3 E3 ligase and promote malignancy. Proc Natl Acad Sci U S A, 2008. 105(36): p. 13568-73.

14. Tong, K.I., et al., Keap1 Recruits Neh2 through Binding to ETGE and DLG Motifs: Characterization of the Two-Site Molecular Recognition Model. Molecular and Cellular Biology, 2006. 26(8): p. 2887-2900.

15. Tong, K.I., et al., Different electrostatic potentials define ETGE and DLG motifs as hinge and latch in oxidative stress response. Mol Cell Biol, 2007. 27(21): p. 7511-21.

16. Karunatilleke, N.C., et al., Nrf2, the Major Regulator of the Cellular Oxidative Stress Response, is Partially Disordered. International Journal of Molecular Sciences, 2021. 22(14): p. 7434.

17. Dunker, A.K., et al., Intrinsically disordered protein. Journal of Molecular Graphics and Modelling, 2001. 19(1): p. $26-59$.

18. Dyson, H.J. and P.E. Wright, Intrinsically unstructured proteins and their functions. Nat Rev Mol Cell Biol, 2005. 6(3): p. 197-208.

19. Uversky, V.N., Intrinsically Disordered Proteins and Their "Mysterious" (Meta)Physics. Frontiers in Physics, 2019. 7(10).

20. Dobson, C.M., Protein folding and misfolding. Nature, 2003. 426(6968): p. 884-890.

21. Wise-Scira, O., et al., Structures of the E46K mutant-type $\alpha$-synuclein protein and impact of E46K mutation on the structures of the wild-type $\alpha$-synuclein protein. ACS chemical neuroscience, 2013. 4(3): p. 498-508.

22. Xue, B., et al., Intrinsically disordered regions of p53 family are highly diversified in evolution. Biochimica et biophysica acta, 2013. 1834(4): p. 725-738.

23. Stadtman, E.R., Oxidation of free amino acids and amino acid residues in proteins by radiolysis and by metal-catalyzed reactions Annu Rev Biochem, 1993. 62: p. 797-821.

24. Dean, R.T., C.R. Roberts, and W. Jessup, Fragmentation of extracellular and intracellular polypeptides by free radicals. Prog Clin Biol Res, 1985. 180: p. 341-50.

25. Davies, K.J., Protein damage and degradation by oxygen radicals. I. general aspects. J Biol Chem, 1987. 262(20): p. 9895-901.

26. Davies, K.J., M.E. Delsignore, and S.W. Lin, Protein damage and degradation by oxygen radicals. II. Modification of amino acids. J Biol Chem, 1987. 262(20): p. 9902-7.

27. Di Simplicio, P., et al., Thiolation and nitrosation of cysteines in biological fluids and cells. Amino Acids, 2003. 25 (3): p. 323-339.

28. Ishida, T. and K. Kinoshita, PrDOS: prediction of disordered protein regions from amino acid sequence. Nucleic Acids Res, 2007. 35(Web Server issue): p. W460-4.

29. Xue, B., et al., PONDR-FIT: a meta-predictor of intrinsically disordered amino acids. Biochimica et biophysica acta, 2010. 1804(4): p. 996-1010.

30. Mészáros, B., G. Erdős, and Z. Dosztányi, IUPred2A: context-dependent prediction of protein disorder as a function of redox state and protein binding. Nucleic Acids Research, 2018. 46(W1): p. W329-W337.

31. The Universal Protein Resource (UniProt), in Nucleic Acids Research. 2008.

32. Kumar, S., et al., MEGA X: Molecular Evolutionary Genetics Analysis across Computing Platforms. Mol Biol Evol, 2018. 35(6): p. 1547-1549.

33. Thomas, B.J. and R. Rothstein, Elevated recombination rates in transcriptionally active DNA. Cell, 1989. 56(4): p. 619-630.

34. Gietz, R.D. and R.H. Schiestl, High-efficiency yeast transformation using the LiAc/SS carrier DNA/PEG method. Nature Protocols, 2007. 2: p. 31.

35. Petropavlovskiy, A.A., et al., A Quantitative Imaging-Based Protocol for Yeast Growth and Survival on Agar Plates. STAR Protocols, 2020: p. 100182. 
36. McMahon, M., et al., Keap1 perceives stress via three sensors for the endogenous signaling molecules nitric oxide, zinc, and alkenals. Proceedings of the National Academy of Sciences, 2010. 107(44): p. 18838-18843.

37. Li, X., et al., Crystal structure of the Kelch domain of human Keap1. J Biol Chem, 2004. 279(52): p. 54750-8.

38. Miseta, A. and P. Csutora, Relationship between the occurrence of cysteine in proteins and the complexity of organisms. Mol Biol Evol, 2000. 17(8): p. 1232-9.

39. Nam, L.B. and Y.-S. Keum, Binding partners of NRF2: Functions and regulatory mechanisms. Archives of Biochemistry and Biophysics, 2019. 678: p. 108184.

40. Uversky, V.N., Intrinsically disordered proteins may escape unwanted interactions via functional misfolding. Biochimica et Biophysica Acta (BBA) - Proteins and Proteomics, 2011. 1814(5): p. 693-712.

41. He, X. and Q. Ma, NRF2 cysteine residues are critical for oxidant/electrophile-sensing, Kelch-like ECH-associated protein-1dependent ubiquitination-proteasomal degradation, and transcription activation. Mol Pharmacol, 2009. 76(6): p. $1265-78$.

42. Fourquet, S., et al., Activation of NRF2 by nitrosative agents and H2O2 involves KEAP1 disulfide formation. J Biol Chem, 2010. 285(11): p. 8463-71.

43. Sun, Z., et al., KPNA6 (Importin $\alpha 7$ )-Mediated Nuclear Import of Keap1 Represses the Nrf2-Dependent Antioxidant Response. Molecular and Cellular Biology, 2011. 31(9): p. 1800-1811.

44. Kumari, S., et al., Reactive Oxygen Species: A Key Constituent in Cancer Survival. Biomarker insights, 2018. 13: p. 1177271918755391.

45. Taguchi, K., et al., Keap1 degradation by autophagy for the maintenance of redox homeostasis. Proceedings of the National Academy of Sciences, 2012. 109(34): p. 13561. 